\title{
Pteridophytes OF ThORANGTLANG WildLIFE SANCTUARY, MIZORAM, INDIA
}

\author{
H.A. Barbhuiya ${ }^{1} \&$ S.K. Singh ${ }^{2}$ \\ 1,2 Botanical Survey of India, Eastern Regional Centre, Shillong, Meghalaya 793003, India \\ ${ }^{1}$ hussainbsi@yahoo.com (corresponding author), ${ }^{2}$ sksbsinc@rediffmail.com
}

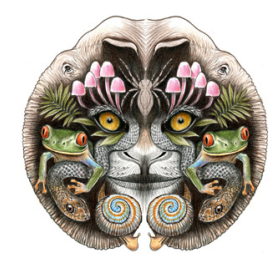

ISSN

Online 0974-7907

Print 0974-7893

OPEN ACCESS
Abstract: A preliminary study on the pteridophytic flora of Thorangtlang Wildlife Sanctuary, Mizoram, India has been made. A total of 36 taxa of pteridophytes are recorded and enumerated for the first time. A brief taxonomic description of each species and the distribution within northeastern India is provided.

Keywords: Fern, fern-allies, Mizoram, Pteridophytes, Thorangtlang.

The Thorangtlang Wildlife Sanctuary (TWS) is one of the most remote and inaccessible localities, situated about $230 \mathrm{~km}$ south-west of Aizawl in Lunglei District of Mizoram, India. It spreads over $50 \mathrm{~km}^{2}$ with an altitude of $800-1380 \mathrm{~m}$. The annual rainfall ranges between 1700-3900 $\mathrm{mm}$ and the average temperature ranges from $8-20{ }^{\circ} \mathrm{C}$ during winter and $19-29{ }^{\circ} \mathrm{C}$ during summer. The area is humid in summer but the months of March and April are comparatively drier. The sanctuary harbors unique natural features of subtropical evergreen and semi-evergreen forests and supports a diversified flora. Actinodaphne macroptera Miq., Dipterocarpus turbinatus C.F. Gaertn, Duabanga grandiflora (DC.) Walp., Garcinia cowa Roxb. ex Choisy, Mallotus roxburghianus Müll. Arg., Mesua ferrea L., Mitragyna diversifolia (Wall. ex G. Don) Havil., Sterculia villosa Roxb., Toona ciliata M. Roem. etc., are the main tree species forming the vegetation of the area.

As far as studies on the pteridophytes of Mizoram is concerned, it appears that this state has received less attention and is known only through sporadic reports by Gage (1901), Fischer (1938), Chandra \& Chandra (1983), Deb \& Dutta (1987) and Benniamin (2011, 2012). Many areas of the state are still unexplored which require systematic exploration.

During a recent field trip to TWS in November 2011, the authors had collected samples of pteridophytic taxa from various habitats. Study of these specimens revealed the occurrence of 36 taxa (28 terrestrial, 7 lithophytic, 1 epiphytic) belonging to 19 genera and 15 families of pteridophytes. All of them are reported for the first time from this sanctuary. The species like Adiantum philippense L., Blechnum orientale L., Thelypteris nudata (Roxb.) C.V.Morton, Leptochilus pteropus (Blume) FraserJenk. and Polystichum pseudotsus-simense Ching grow luxuriantly in the study area.

The families are enumerated in text according to Fraser-Jenkins (2009). However, the genera and species within the families are listed alphabetically. The authorities of plant names follow Brummitt \& Powell (1992) while the taxonomic citation is based on published literature and IPNI, Tropicos and The Plant List. The studied specimens are deposited in the Cryptogamic Section of Herbarium of Botanical Survey of India, Shillong (ASSAM) (Appendix 1).

DOI: http://dx.doi.org/10.11609/JoTT.03311.6249-68

Editor: S.C. Verma, Professor Emeritus, Panjab University, Chandigarh, India.

Date of publication: 26 August 2014 (online \& print)

Manuscript details: Ms \# 03311 | Received 18 August 2012 | Final received 07 August 2014 | Finally accepted 10 August 2014

Citation: Barbhuiya, H.A. \& S.K. Singh (2014). Pteridophytes of Thorangtlang Wildlife Sanctuary, Mizoram, India. Journal of Threatened Taxa 6(9): 6249-6268; http://dx.doi.org/10.11609/JoTT.03311.6249-68

Copyright: (C Barbhuiya \& Singh 2014. Creative Commons Attribution 4.0 International License. JoTT allows unrestricted use of this article in any medium, reproduction and distribution by providing adequate credit to the authors and the source of publication.

Funding: Though there is external funding, the materials were collected during execution of AAP Project of BSI, ERC, Shillong.

Competing Interest: The authors declare no competing interests.

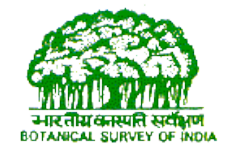

Acknowledgements: Authors are grateful to the Director, Botanical Survey of India, Kolkata and Head, Eastern Regional Centre, BSI, Shillong for facilities and encouragements and to the officials of Forest Department of Mizoram for rendering the logistic support during field exploration. Sincere thanks are also extended to Dr B.S. Kholia, Dehradun and C.R. Fraser-Jenkins, Kathmandu for their help in identification of a few species. 


\section{TAXONOMIC ENUMERATION}

\section{Selaginellaceae}

1. Selaginella chrysorrhizos Spring, Mém. Acad. Sci. Bélg. 24 : 251.1850.

Stem erect; lateral leaves oblong-lanceolate, entire at base, median leaves aristate, white margined ovate, elliptic, or lanceolate; arista up to half as long as lamina. Sporophylls dimorphic, ciliate.

Specimen examined: 124397, 30.xi.2011, terrestrial, TWS, coll. S.K. Singh \& H.A. Barbhuiya.

Distribution: Assam (Alston 1945), Meghalaya (Bir et al. 1989), Manipur, Mizoram, Sikkim (Ghosh et al. 2004) and in other parts of India and the world.

2. Selaginella decipiens Warb., Monsunia 1: 127. 1899.

Stem erect rooting at base only; median leaves aristate, arista less than half as long as the lamina. Sporophylls dimorphic, not ciliate.

Specimen examined: 124295, 30.xi.2011, terrestrial, TWS, coll. S.K. Singh \& H.A. Barbhuiya.

Distribution: Arunachal Pradesh (Singh \& Panigrahi 2005), Assam (Alston 1945), Meghalaya (Baishya \& Rao 1982), Mizoram (Fischer 1938) and in other parts of India and the world.

3. Selaginella involvens (Sw.) Spring, Bull. Acad. Royl. Sci. Bruxelles 10: 136. 1843. Lycopodium involvens Sw., Syn. Fil., 182. 1806.

Stem very densely tufted; leaves of the lower plane very crowded, contiguous, ascending, median leaves ovate, acute. Sporophylls monomorphic, margin denticulate.

Specimen examined: 124297, 30.xi.2011, terrestrial, TWS, coll. S.K. Singh \& H.A. Barbhuiya.

Distribution: Arunachal Pradesh (Singh \& Panigrahi 2005), Meghalaya (Baishya \& Rao 1982), Manipur, Sikkim (Alston 1945), Mizoram (Gage 1901 as Selaginella caulescens (Wall. ex Hook. \& Grev.) Spring) and in other parts of India and the world.

Note: It is very similar to S. bryopteris (L.) Baker [commonly known as Sanjeevani], commonly found in the plains areas of the country. But in having short acuminated apex of median leaves which margin is denticulated throughout and spores with perispore, it can be differentiated from the latter ones easily.

\section{Lygodiaceae}

4. Lygodium salicifolium C. Presl, Suppl. Tent. Pterid. 102. 1845. (Image 1)

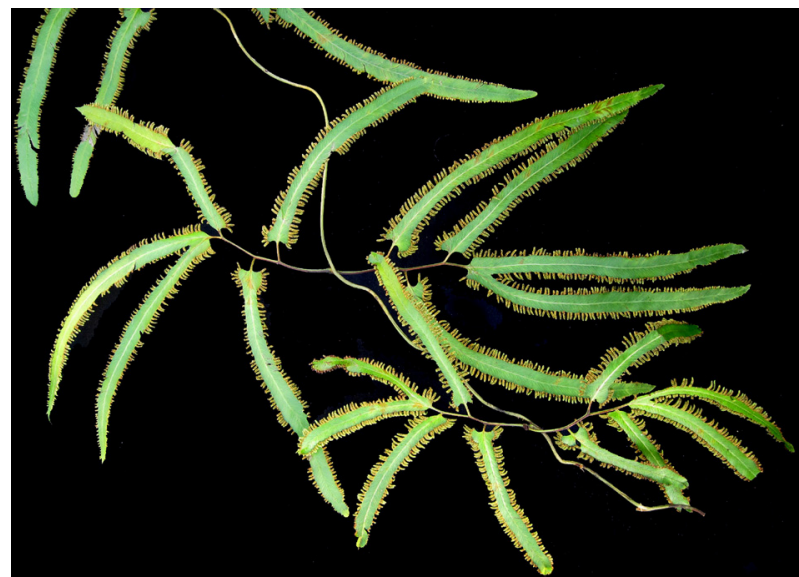

Image 1. Lygodium salicifolium. (c) Authors

Rhizome short creeping. Fronds up to $5 \mathrm{~m}$ long; secondary rachis pinnate - bipinnate, all pinnules equal in size, base wide cuneate to cordate or auricled, terminal pinnae similar to lateral, in some cases basal pinnules of secondary pinnae lobed - pinnate; mid veins hairy, veins once or twice forked; dormant apex hairs without swollen bases; costules more or less raised at mid lamina.

Specimen examined: 124275, 30.xi.2011, terrestrial, TWS, coll. S.K. Singh \& H.A. Barbhuiya.

Distribution: Assam, Meghalaya, Arunachal Pradesh, Tripura (Singh \& Panigrahi 1984) and in other parts of India and the world.

Note: Closely allied with L. floxuosum (L.) Sw., however, the latter differs in having largest basal pinnules and the succeeding ones gradually reduced; basal pinnules stalked becoming sessile and subsessile and thereafter adnate upwards (see Singh \& Panigrahi 1984). This is reported here for the first time from Mizoram.

\section{Polypodiaceae}

5. Drynaria coronans (Wall. ex Mett.) J. Sm. ex T. Moore, Index Fil. 345. 1862. Polypodium coronans Wall. ex Mett., Abh. Senckenberg. Naturf. Ges. 1: 121. 1856.

Rhizome creeping, thick, densely scaly throughout. Fronds sessile, lobed almost to rachis, lamina stiffly coriaceous, the base of lamina broadly rounded, lobes of the upper part of laminae ascending, usually more than a dozen pairs of pinna-like lobes, linear-subtriangular, attenuately acuminate at apex, entire at margin. Sori one or very rarely two rows between main veins, more or less elongate.

Specimen examined: 124287, 30.xi.2011, epiphytic, TWS, coll. S.K. Singh \& H.A. Barbhuiya. 
Distribution: Arunachal Pradesh (Singh \& Panigrahi, 2005 as Aglaomorpha coronans (Wall.) Copel.), Assam (Borthakur et al. 2001), Meghalaya (Baishya \& Rao 1982 as Pseudodrynaria coronans (Wall. ex Mett.) Ching), Tripura (Deb 1981, as P. coronans), Mizoram (Fischer 1938), Nagaland (Jamir \& Rao 1988) and in other parts of the world.

6. Leptochilus pteropus (Blume) Fraser-Jenk., Taxon. Revis. Indian Subcontinental Pteridophytes 62. 2008. Polypodium pteropus Blume, Enum. Pl. Javae 125, add. 3. 1828.

Fronds simple to trifoliate, simple one broadest in the middle, narrowing towards the attenuate base, longacuminate at apex, entire, the lateral lobes of trifoliate laminae variable in size. Stipe and lower rachis \pm densely clothed with small dark scales. Sori round to more or less elongate, many, irregularly scattered on the under surface of laminae.

Specimen examined: 124290, 30.xi.2011, lithophytic, TWS, coll. S.K. Singh \& H.A. Barbhuiya.

Distribution: Arunachal Pradesh (Singh \& Panigrahi 2005), Assam (Borthakur et al. 2001), Meghalaya (Baishya \& Rao 1982 as Kaulinia pteropus (Blume) B.K. Nayar), Mizoram (Fischer 1938), Nagaland (Jamir \& Rao 1988 as K. pteropus), Sikkim (Beddome 1892 as Pleopeltis pteropus (Blume) Bedd.), Manipur, Tripura (Ghosh et al. 2004) and in other parts of India and the world.

\section{Cyatheaceae}

7. Cyathea brunoniana (Wall. ex Hook.) C.B. Clarke \& Baker, J. Linn. Soc., Bot. 24. 409. 1888. Alsophila brunoniana Wall. ex Hook., Sp. Fil. 1: 52. 1844.

Fronds bipinnate to tripinnatifid, glaucous beneath. Stipe rachis and costa spine less, pale brown, glossy; segment with few or no teeth. Sori exindusiate, spherical, occurring near costules, brown in colour; fertile segments narrower than the sterile ones.

Specimen examined: 126003, 30.xi.2011, terrestrial, TWS, coll. S.K. Singh \& H.A. Barbhuiya.

Distribution: Arunachal Pradesh (Dixit 1998 as Sphaeropteris brunoniana (Wall. ex Hook.) Tryon), Manipur, Meghalaya (Kachroo et al. 1989), Mizoram (Kumar et al. 2012), Nagaland (Jamir \& Rao 1988), Sikkim (Kholia 2010) and in other parts of India and the world.

8. Cyathea gigantea (Wall. ex Hook.) Holttum, Gard. Bull. Straits Settlem. 8: 318. 1935. Alsophila gigantea Wall. ex Hook., Sp. Fil. 1: 53. 1844. (Image 2)

Trunk massive erect, ca. 2-4 m tall. Lamina bipinnate, deltoid, dark green. Rachis sparsely scaly. Veins simple

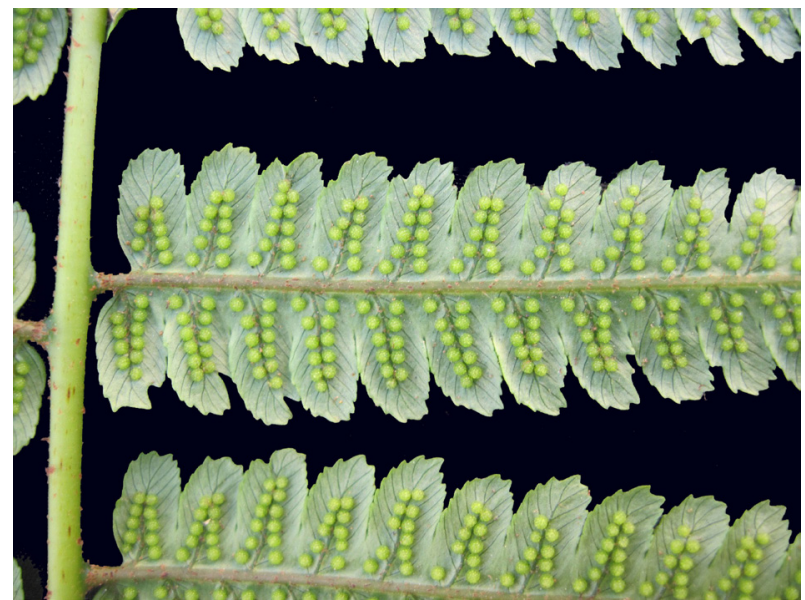

Image 2. Cyathea gigantea. (C) Authors

not forked. Costae and costules minutely scaly. Sori arranged in ' $\mathrm{V}$ ' shape in each lobe, exindusiate; sporangia numerous compact.

Specimen examined: 124277, 30.xi.2011, terrestrial, TWS, coll. S.K. Singh \& H.A. Barbhuiya.

Distribution: Arunachal Pradesh (Singh \& Panigrahi, 2005), Assam (Borthakur et al. 2001), Manipur (Malick \& Safui 1987), Meghalaya (Baishya \& Rao 1982), Nagaland (Jamir \& Rao, 1988), Sikkim (Kholia 2010), Tripura (Deb 1981) and in other parts of India and the world.

\section{Dennstaedtiaceae}

9. Microlepia calvescens (Wall. ex Hook.) C. Presl, Epimel. Bot. 95. 1851. Davallia calvescens Wall. ex Hook., Sp. Fil. 1: 172, t. 48B. 1846.

Stipe erect to $30 \mathrm{~cm}$ long; fronds 1-pinnate, ca. $50 \mathrm{~cm}$ long, both surfaces glabrous; rachis and costae villous; pinnae pinnatified, lathery, shortly stalked. Sori placed at the end of the veins; indusium glabrous.

Specimen examined: 124281, 30.xi.2011, terrestrial, TWS, coll. S.K. Singh \& H.A. Barbhuiya.

Distribution: Arunachal Pradesh, Manipur, Meghalaya, Mizoram (Fraser-Jenkins 2012) and in other parts of the world.

10. Microlepia speluncae (L.) T. Moore, Index Fil. 93. 1857. Polypodium speluncae L., Sp. PI. 1093. 1753. (Image 3)

Lamina large tripinnate to quadripinnatifid, ovateoblong, both surfaces sparsely hairy; rachis stramineous to brownish, grooved on upper surface, more or less hairy. Pinnules broadly lanceolate, pinnatisect almost to rachillae, base unequal, cuneate basiscopically, subtruncate acroscopically, apex acuminate. Sori a 


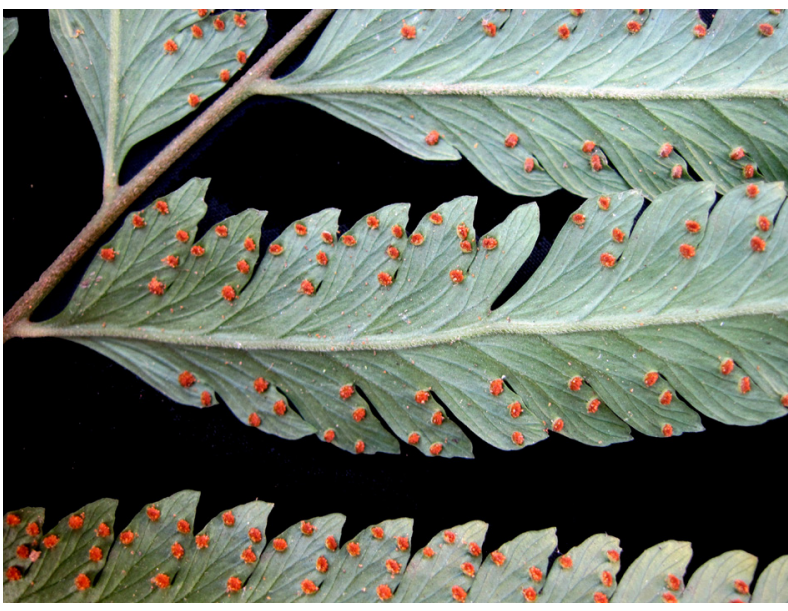

Image 3. Microlepia calvescens. (C) Authors

little within the margin of lobes, small; indusia saucershaped, hairy.

Specimen examined: 124296, 124272, 124274, 30.xi.2011, terrestrial, TWS, coll. S.K. Singh \& H.A. Barbhuiya.

Distribution: Arunacahl Pradesh (Singh \& Panigrahi 2005), Assam (Borthakur et al. 2001), Manipur (Ghosh et al. 2004), Meghalaya (Beddome 1892), Mizoram (Fischer 1938), Nagaland (Jamir \& Rao 1988), Tripura (Deb 1981) and in other parts of India and the world.

11. Pteridium revolutum (Blume) Nakai, Bot. Mag. (Tokyo) 39(461): 109. 1925. Pteris revoluta Blume, Enum. PI. Javae 2: 214. 1828.

Fronds subcoriaceous, margins often revolute; lamina 3-pinnate-pinnatifid, broadly triangular or ovatetriangular. Pinnae 4-6 pairs, opposite, decumbent, oblong, base subtruncate, basal pinnae 2-pinnate. Rachises, costae, and costules with pallid or light brown hairs or verrucose, glabrescent. Secondary pinnae lanceolate, up to $8 \times 1.5 \mathrm{~cm}$

Specimen examined: 124280, 30.xi.2011, terrestrial, TWS, coll. S.K. Singh \& H.A. Barbhuiya.

Distribution: Arunachal Pradesh (Benniamin 2010), Manipur (Shukla \& Baishya 1980 as Pteridium aquilinum (L.) Kuhn), Mizoram (Fischer 1938 as P. aquilinum), Nagaland (Jamir \& Rao 1988 as P. aquilinum), Meghalaya (Baishya \& Rao 1982 as $P$. aquilinum) and in other parts of India and the world.

\section{Lindsaeaceae}

12. Lindsaea ensifolia Sw., J. Bot. (Schrader) 1800(2): 77. 1801.

Laminae simply pinnate, lateral pinnae 3-7 pairs, linear lanceolate, acute at apex, cuneate, rounded or subtruncate at base, margins entire. Sori continuous along margin; indusia firm, nearly reaching the edges.

Specimen examined: 124394, 30.xi.2011, terrestrial, TWS, coll. S.K. Singh \& H.A. Barbhuiya.

Distribution: Arunachal Pradesh (Singh \& Panigrahi 2005), Assam (Borthakur et al. 2001), Manipur (Clarke 1890), Meghalaya (Baishya \& Rao 1982), Mizoram (Bir et al. 1991), Nagaland (Jamir \& Rao 1988), Tripura (Kachroo et al. 1989), Sikkim (Clark 1880; Beddome 1892 as Schizoloma ensifolium (Sw.) J. Sm.) and in other parts of India and the world.

\section{Pteridaceae}

13. Pityrogramma calomelanos (L.) Link, Handbuch 3: 20. 1833. Acrostichum calomelanos L., Sp. PI. 1072. 1753.

Stipes dark black, polished, scaly on lower part, glabrous upwards, covered with white powder at the young stage. Lamina triangular to oblong, with acuminate apex, bipinnate-tripinnatifid, 15-30 × 8-15 $\mathrm{cm}$, white powdery beneath; rachis grooved on upper surface. Sporangia placed along veins throughout the lower surface, without any protection.

Specimen examined: 124279, 30.xi.2011, terrestrial, TWS, coll. S.K. Singh \& H.A. Barbhuiya.

Distribution: Arunachal Pradesh (Singh \& Panigrahi, 2005), Assam (Borthakur et al. 2001), Manipur, Meghalaya (Baishya \& Rao 1982), Mizoram (Deb \& Dutta 1987), Nagaland (Jamir \& Rao 1988), Tripura (Deb 1981), Sikkim (Panigrahi 1975) and in other parts of India and the world.

Note: It is noteworthy to mention here that $P$. calomelanos is a native of tropical America and a well known silver fern of horticulture, and has escaped cultivation by getting naturalized in all warm lands including India (Verma 1966). Although it was collected by H.F. Blanford in 1866 from Mercara, Coorg (S. India) and in 1908 by W.G. Craib from Mungpoo (E. Himalaya), the first published record of the species in the wild state from India is that of Blatter \& d'Almeida (1922) from Bombay Presidency, and of Mehra (1932) from Sikkim. In Darjeeling and Sikkim Himalaya it is common at low altitudes.

14. Pteris biaurita L. subsp. walkeriana FraserJenk. \& Rajkumar, Taxon. Revis. Indian Subcontinental Pteridophytes 115. 2008.

Stipe straw-colored. Lamina pinnate, hard papery; costa with spines along adaxial groove. Pinnae opposite or nearly so, up to 12 pairs, straight, ascending, linearlanceolate, broadly cuneate at base, gradually narrowing 
towards acuminate apex, deeply lobed towards costa, basal pinnae bearing a long basiscopic pinnule just like lateral ones. Venation areolate, opposite basal veins anastomosing, forming an elevated triangular arch along costa or sometimes free. Sori marginal, usually continuous along segments except at bottom of sinus and at apex; indusia thin, pale.

Specimen examined: 124276, 124398, 30.xi.2011, terrestrial, TWS, coll. S.K. Singh \& H.A. Barbhuiya.

Distribution: Arunachal Pradesh, Assam, Manipur, Meghalaya, Nagaland, Sikkim (see Fraser-Jenkins 2008) and in other parts of India and the world.

15. Pteris khasiana (C.B.Clarke) Hieron., Hedwigia 55: 364. 1914. Pteris quadriaurita Retz. var. khasiana C.B.Clarke Trans. Linn. Soc. London, Bot. 1(7): 466, t. 53. 1880. (Image 4)

Stipe greenish-brown, without scales. Lamina light green, 2-pinnate, ovate to ovate-triangular; lateral pinnae 7-8 pairs, mostly opposite, basal pinnae with one or two developed basal basicopic pinnules; segments 15-28 pairs, oblong, slightly falcate, alternate or opposite, conjoined or 1-2 mm apart, apex obtuse, basiscopic segments longer than acroscopic segments; veins conspicuous on both surfaces, oblique forked at the base.

Specimen examined: 124393, 30.xi.2011, terrestrial, TWS, coll. S.K. Singh \& H.A. Barbhuiya.

Distribution: Mizoram, Meghalaya (Clarke 1880) and in other parts of India and the world.

16. Pteris pellucida C. Presl, Reliq. Haenk. 1(1): 55. 1825.

Fronds pinnate, stipe straw-colored to light brown, rachis slightly winged, pinnae lanceolate, sessile, margins minutely undulate, subcartilaginous, acuminate; veins simple or 2-forked, sori marginal.

Specimen examined: 124294, 30.xi.2011, terrestrial, TWS, coll. S.K. Singh \& H.A. Barbhuiya.

Distribution: Arunachal Pradesh (Singh \& Panigrahi 2005), Assam (Borthakur et al. 2001), Mizoram (Fischer 1938), Nagaland (Jamir \& Rao 1988), Sikkim (Clark 1880), and in other parts of India and the world.

\section{Adiantaceae}

17. Adiantum philippense L., Sp. PI. 1094. 1753.

Lateral pinnae large at base, slightly reduced in size upwards, distinctly stalked; leaflets crescent-shaped. Sori at margin of leaflets, reflexed soral flaps elongate, usually 5-8 mm long, but up to $1.5 \mathrm{~cm}$.

Specimen examined: 124299, 30.xi.2011, terrestrial,

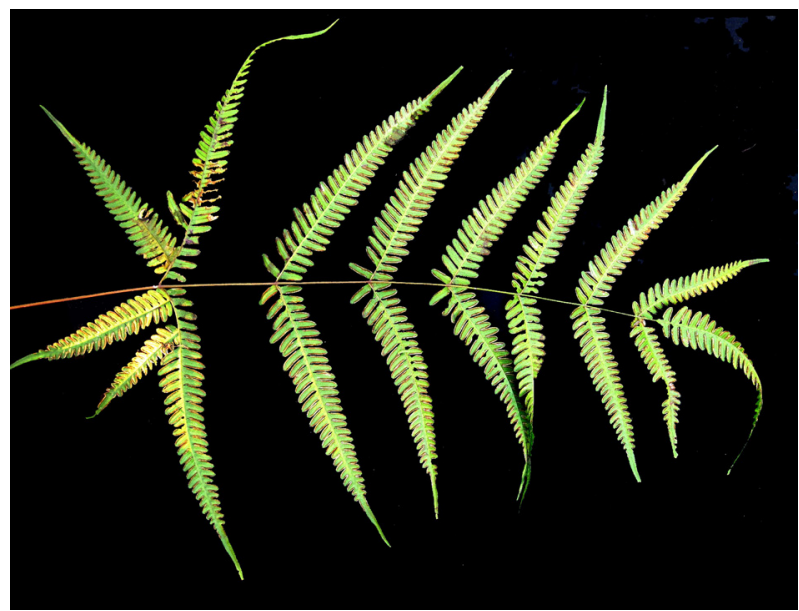

Image 4. Pteris khasiana. (C) Authors

TWS, coll. S.K. Singh \& H.A. Barbhuiya.

Distribution: Arunachal Pradesh (Singh \& Panigrahi, 2005), Assam (Borthakur et al. 2001), Meghalaya (Baishya \& Rao 1982), Mizoram (Fischer 1938 as A. lunulatum Burm f.), Nagaland (Jamir \& Rao 1988), Sikkim (Kholia, 2010), Tripura (Deb 1981) and in other parts of India and the world.

Note: The species was previously known as $A$. lunulatum (Beddome 1863, 1892; Mehra 1939), but subsequently it was reduced to the synonymy of Adiantum philippense. Verma (1962 '1961'), in order to resolve the identity of the taxa within the discovered species complex, argued in favour of the nomenclature A. Iunulatum Burm. f. More recently, based on the successful search of Kamel's original specimen of Adiantum philippense at BM, from the Philippines, Verma \& Fraser-Jenkins (2008) not only concluded the correct name of the taxon as Adiantum philippense, but also dealt with the taxonomic treatment of the various cytotypes from the eastern Himalaya, in particular recognizing subspecies within it (see Verma \& FraserJenkins 2008).

\section{Vittariaceae}

18. Antrophyum coriaceum (D. Don) Wall. ex T. Moore, Index Fil. 80. 1858. Hemionitis coriacea D. Don Prodr. Fl. Nepal. 13. 1825. (Image 5)

Rhizome slender, short creeping; fronds clustered; stipes indistinct; laminae subcoriaceous, linearlanceolate, $5-13 \times 0.8-1.5 \mathrm{~cm}$, widest at middle, apex narrowly acute or caudate; costa indistinct; veins reticulate, raised above, invisible below. Soral lines 3-5, zigzag, subparallel, continuous or interrupted; paraphyses filiform. 


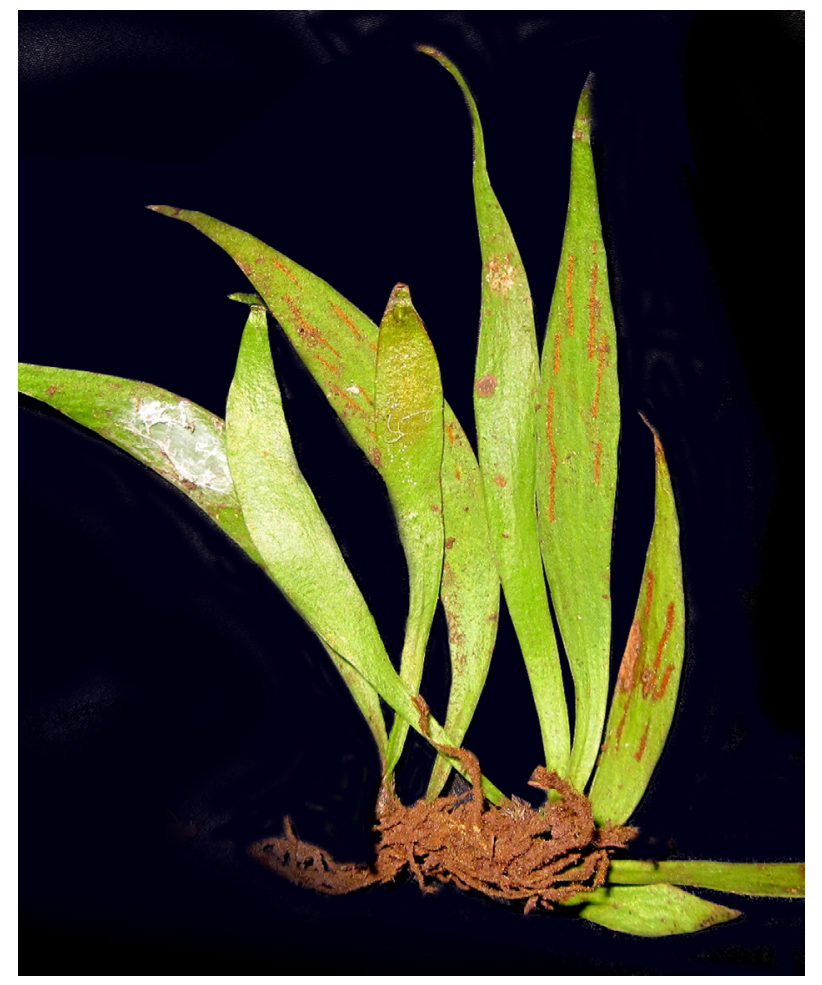

Image 5. Antrophyum coriaceum. (C) Authors

Specimen examined: 124399, 30.xi.2011, lithophytic, TWS, coll. S.K. Singh \& H.A. Barbhuiya.

Distribution: Mizoram, Arunachal Pradesh, Sikkim and in other parts of the world.

\section{Aspleniaceae}

19. Asplenium finlaysonianum Wall. ex Hook., Icon. PI. 10: t. 937. 1854.

Pinnae 2-6 on each side, stalked, longest pinnae at base, narrowly ovate to elliptic, mostly asymmetrical and cuneate at base, long acuminate to caudate at apex, terminal pinna rhomboid or somewhat trifid. Veins free, branched, more or less connected near margin. Sori linear, oblique to costae.

Specimen examined: 124291, 30.xi.2011, lithophytic, TWS, coll. S.K. Singh \& H.A. Barbhuiya.

Distribution: Arunachal Pradesh (Singh \& Panigrahi 2005), Assam (Borthakur et al. 2001), Manipur (Clark 1890), Meghalaya (Baishya \& Rao 1982), Nagaland (Jamir \& Rao 1988), Mizoram, Sikkim, West Bengal (Bir 1998) and in other parts of India and the world.

20. Asplenium obscurum Blume, Enum. Pl. Javae 2: 181. 1828.

Frond pinnate, rachis abaxially dull, greyish green to brown. Pinnae usually more than 15 pairs, simple, dimidiate, marginal teeth entire, veins ending just below marginal teeth. Sorus medial or inframedial, with a single or double indusium, overlying sorus.

Specimen examined: 124300, 30.xi.2011, terrestrial, TWS, coll. S.K. Singh \& H.A. Barbhuiya.

Distribution: Arunachal Pradesh (Singh \& Panigrahi 2005 as Asplenium excisum C. Presl), Meghalaya (Vasudeva et al. 1990) and in other parts of India and the world.

21. Asplenium phyllitidis D.Don, Prodr. Fl. Nepal. 7. 1825.

Frond simple, long, up to $10 \mathrm{~cm}$ broad at middle, midrib obtusely keeled abaxially, gradually narrowing towards both apex and base, veins forked, parallel. Sori linear, born on acroscopic of veinlets from base to more than half way towards margin; indusial linear, membranous, entire, persistent.

Specimen examined: 124292, 30.xi.2011, lithophytic, TWS, coll. S.K. Singh \& H.A. Barbhuiya.

Distribution: Arunachal Pradesh, Assam, Manipur (Fraser-Jenkins 2008), Sikkim (Kohlia 2010), Meghalaya (Baishya \& Rao 1982), Mizoram (Benniamin 2012), Nagaland (Jamir \& Rao 1988) and in other parts of India and the world.

\section{Thelypteridaceae}

22. Thelypteris clarkei (Bedd.) C.F. Reed in Phytologia 17: 268. 1968. Pleocnemia clarkei Bedd., Ferns Brit. India 15. 1876.

Rhizome creeping, scales narrow, brown, hairy. Laminae deltate or oblong-lanceolate, acute at apex, basal pair of pinnae largest, lower 1 or 2 pairs deflexed, pinnae narrowed at base, middle pinnae patent or ascending, more or less falcate, caudate-acuminate at apex, truncate at sessile base, deeply lobed nearly to costa; rachis and costa rather densely hairy throughout, one pair of opposite veinlets anastomosing beneath the sinus. Sori medial; indusia small, persistent, shortly hairy

Specimen examined: 126002, 30.xi.2011, terrestrial, TWS, coll. S.K. Singh \& H.A. Barbhuiya.

Distribution: Arunachal Pradesh (Singh \& Panigrahi 2005 as Cyclosorus cylindrothrix (Rosenst.) Ching), Assam (Borthakur et al. 2001 as Christella cylindrothrix (Rosenst.) Holttum), Meghalaya (Baishya \& Rao 1982 as C. parasiticus (L.) Lev.), Mizoram (Benniamin 2012), Sikkim (Beddome 1892; Dixit 1984 as Christella clarkei (Bedd.) Holttum) and in other parts of the world.

Note: In India, this species was earlier known as Thelypteris (Christella) cylindothrix, but FraserJenkins (1997) properly identified it and solved the 
nomenclatural problem.

23. Thelypteris crinipes (Hook.) K.Iwats., J. Jap. Bot. 38: 315. 1963. Nephrodium crinipes Hook., Sp. Fil. 4: 71. 1862.

Stipe and rachis densely scaly throughout. Lamina oblong-lanceolate; several lower pinnae distinctly reduced and immersed among scales, lower normal pinnae straight, ascending, linear, gradually narrowing towards long-acuminate apex. Veins 8-10 pairs, lower pairs anastomosing. Sori medial or nearer to margin, round; indusia round-reniform, densely hairy.

Specimen examined: 126001, 30.xi.2011, terrestrial, TWS, coll. S.K. Singh \& H.A. Barbhuiya.

Distribution: Arunachal Pradesh (Singh \& Panigrahi, 2005 as Cyclosorus crinipes (Hook.) Ching), Assam (Borthakur et al. 2001 as Christella crinipes (Hook.) Holttum), Meghalaya, (Kachroo et al. 1989 as Christella crinipes), Nagaland (Clarke 1890 as Nephrodium crinipes), Sikkim (Hooker 1862), Tripura (Das 1992 as Christella crinipes) and in other parts of India and the world.

24. Thelypteris evoluta (C.B.Clarke) Tagawa \& K.Iwats. in Acta Phytotax. Geobot. 22: 101. 1967. Nephrodium amboinense var. evolutum C.B.Clarke \& Baker, J. Linn. Soc. Bot. 24: 417. 1888.

Rhizome short creeping. Laminae once-pinnate, lower 2-5 pairs abruptly reduced, strongly auricled. Pinnae widely spaced, ca. 15 pairs, very shallowly lobed, lobes rectangular; 2-3 pairs of opposite veins anastomose below the sinus. Largest pinnae not auricled, sessile, short-acuminate. Sori medial, lower ones divergent; indusia bearing minute hairs as lamina.

Specimen examined: 124288, 30.xi.2011, terrestrial, TWS, coll. S.K. Singh \& H.A. Barbhuiya.

Distribution: Arunachal Pradesh (Singh \& Panigrahi, 2005 as Cyclosorus evolutus (Bedd.) Ching), Assam (Borthakur et al. 2001 as Christella evoluta (C.B. Clarke \& Baker) Holttum), Meghalaya (Baishya \& Rao 1982 as C. evoluta), Mizoram (Benniamin 2012), Nagaland (Beddome 1892 as Nephrodium evolutum Bedd.) and in other parts of the world.

25. Thelypteris hirtisora (C.Chr.) K.Iwats. in J. Jap. Bot. 38: 314. 1963. Dryopteris hirtisora C. Chr. in Contr. U.S. Natl. Herb. 26: 277. 1931.

Rhizomes long creeping, with ovate-lanceolate dark brown scales. Fronds distant, lamina widest towards the base, apices caudate with a large apical pinna. Middle pinnae $1-2 \mathrm{~cm}$ wide; proximal pinnae not decrescent at bases. Laminae papery, brownish green when dried. Pinnae ca. 15 pairs, lobed to about half their depth, with pointed lobes. Veins 8-10 pairs, basal 1-2 pairs anastomosing. Sori orbicular, medial; indusia densely hairy. Sporangia bearing several hairs.

Specimen examined: 124284, 30.xi.2011, terrestrial, TWS, coll. S.K. Singh \& H.A. Barbhuiya.

Distribution: Mizoram, Nagaland (Holttum 1979 as Sphaerostephanos hirtisorus (C.Chr.) Holttum) and in other parts of the world.

26. Thelypteris nudata (Roxb.) C.V.Morton in Contr. U.S. Natl. Herb. 38: 352. 1974. Polypodium nudatum Roxb. in Calcutta J. Nat. Hist. 4: 491. 1844.

Lamina oblong, lateral pinnae lanceolate, sessile, ascending, gradually narrowing towards long-acuminate apex, rounded to narrowly cuneate at base, margin crenate, terminal pinna like lateral ones, rounded to subtruncate at base; marginal lobes rounded to slightly acute at their apices, with a cartilaginous margin. Veins 20-22 pairs, 19 pairs anastomosing with 2-3 terminal free pairs. Sori rather close to excurrent veinlets or medial in two rows between costules; indusia setose, often deciduous.

Specimen examined: 124298, 30.xi.2011, terrestrial, TWS, coll. S.K. Singh \& H.A. Barbhuiya.

Distribution: Arunachal Pradesh (Singh \& Panigrahi 2005 as Pronephrium nudatum), Assam (Borthakur et al. 2001 as P. nudatum), Manipur (Malick \& Safui 1987 as $P$. nudatum), Meghalaya (Baishya \& Rao 1982 as $P$. nudatum), Mizoram (Kachroo et al. 1989 as P. nudatum), Nagaland (Jamir \& Rao 1988 as P. nudatum), Tripura (Das, 1992 as $P$. nudatum) and in other parts of India and the world.

27. Thelypteris tenera (Roxb.) Fraser-Jenk., Taxon. Revis. Indian Subcontinental Pteridophytes 418. 2008. Polypodium tenerum Roxb. Calcutta J. Nat. Hist. 4: 490. 1844.

Rhizome creeping or sub-erect. Stipe $15-25 \mathrm{~cm}$ long. Fronds alternately-pinnate; pinnae linear-lanceolate, obliquely auriculated at base, acuminate, lobed nearly half way to costae. Veins 3-5 pairs in each lobe, simple free. Sori few, on each side of the veins, involucre reniform.

Specimen examined: 124289, 30.xi.2011, terrestrial, TWS, coll. S.K. Singh \& H.A. Barbhuiya.

Distribution: Arunachal Pradesh (Banniamin 2010), Assam (Borthakur et al. 2001 as Trigonospora ciliata (Benth.) Holttum), Meghalaya (Baishya \& Rao 1982 as T. ciliata), Mizoram, Nagaland (Jamir \& Rao 1988 as $T$. 
ciliata), Tripura (Deb 1981 as Thelypteris ciliata (Wall. ex Benth.) Ching) and in other parts of India and the world.

\section{Woodsiaceae}

28. Diplazium pseudosetigerum (Christ) Fraser-Jenk., Indian Fern J. 25: 36. 2008. Athyrium pseudosetigerum Christ, Bull. Acad. Int. Géogr. Bot. Mans. 17: 147. 1907. (Image 6)

Rhizome erect, clumped, brown, densely scaly at apex. Lamina herbaceous, base 3-pinnate, rachis and costa stramineous, glabrous, shallowly grooved adaxially. Secondary pinnule of lower pinna mostly acuminate at apex, serrate at margin. Sori 2 or 3 pairs per lobe, medial, oblong, 1-1.5 mm; indusia light brown, oblong, thinly membranous, vaulted when mature, irregularly ruptured dorsally.

Specimen examined: 124286, 30.xi.2011, terrestrial, TWS, coll. S.K. Singh \& H.A. Barbhuiya.

Distribution: Arunachal Pradesh (Singh \& Panigrahi 2005 as Diplazium axillare Ching), Assam, Manipur (Fraser-Jenkins 2008), Meghalaya (Baishya \& Rao 1982 as Athyrium asperum (Blume) Milde), Nagaland (Jamir \& Rao 1988 as $A$. asperum) and in other parts of the world.

\section{Dryopteridaceae}

29. Polystichum pseudotsus-simense Ching, Bull. Fan Mem. Inst. Biol. 2(9): 190. 1931. (Image 7)

Rhizome short, erect. Stipes green to stramineous, scales at base. Laminae coriaceous, broadly oblong, apex caudate, bipinnate at base, pinnatifid towards apex, ultimate segments subrhomboid to oblong, unequal at base, somewhat serrulate at margin, aristate; veins free, pinnately forked. Sori submarginal, large, indusium

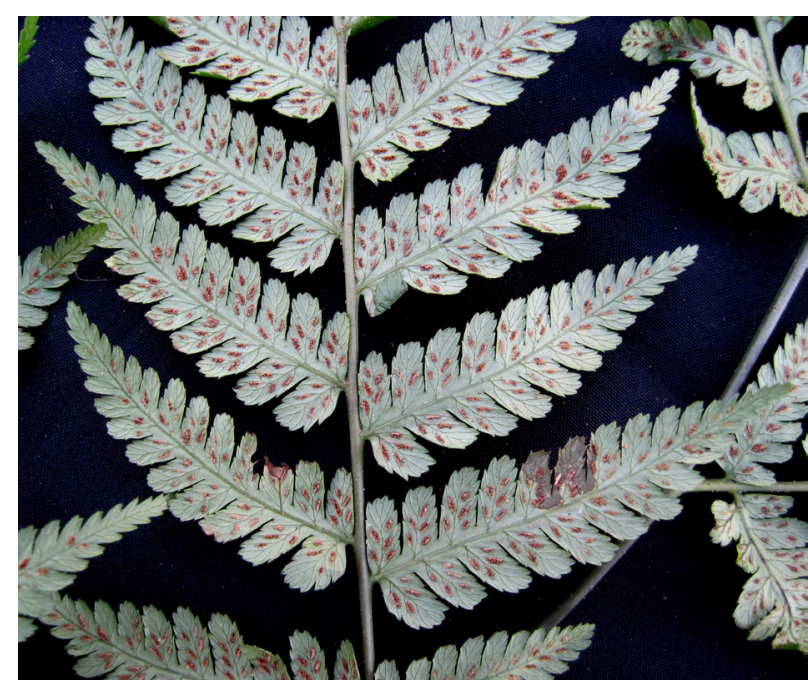

Image 6. Diplazium pseudosetigerum. @ Authors entire.

Specimen examined: 124273, 124282, 30.xi.2011, terrestrial, TWS, coll. S.K. Singh \& H.A. Barbhuiya.

Distribution: Arunachal Pradesh (Singh \& Panigrahi 2005), Meghalaya (Baishya \& Rao 1982 as Polystichum semifertile (C.B. Clarke) Ching), Nagaland (Jamir \& Rao 1988 as $P$. semifertile) and in other parts of India and the world.

30. Tectaria fuscipes (Wall. ex Bedd.) C.Chr., Contr. U.S. Natl. Herb. 26: 290. 1931. Aspidium fuscipes Wall. ex Bedd., Suppl. Ferns Brit. Ind.: 15: t 366. 1876.

Fronds usually dimorphic. Sterile fronds stipe scaly on lower part, pubescent throughout, lamina bipinnatifid, oblong to oblong-lanceolate, acuminate at apex, basal pinnae asymmetrically oblong-subdeltate, caudateacuminate at apex, bearing large basal basiscopic pinnules, fertile frond usually narrower. Sori terminal on acroscopic branches of veinlets, round, irregularly dispersed on lower surface of pinna.

Specimen examined: 124395, 30.xi.2011, terrestrial, TWS, coll. S.K. Singh \& H.A. Barbhuiya.

Distribution: Arunachal Pradesh (Singh \& Panigrahi, 2005), Assam (Borthakur et al. 2001), Meghalaya (Baishya \& Rao 1982 as Tectaria paradoxa (Fee) Sledge), Mizoram (Bir et al. 1991), Nagaland (Jamir \& Rao 1988 as Ctenitopsis fuscipes (Wall. ex Bedd.) C. Chr.), Sikkim (Kholia 2010) and in other parts of the world.

31. Tectaria polymorpha (Wall. ex Hook.) Copel., Philipp. J. Sci., C 2: 413. 1907. Aspidium polymorphum Wall. ex Hook., Sp. Fil. 4: 54. 1862. (Image 8)

Rhizome creeping. Lamina ovate, simply pinnate with ca. 3 pairs of lateral pinnae, pinnae narrowly ovate, base cuneate, apex caudate, shortly stalked, basal pinnae not

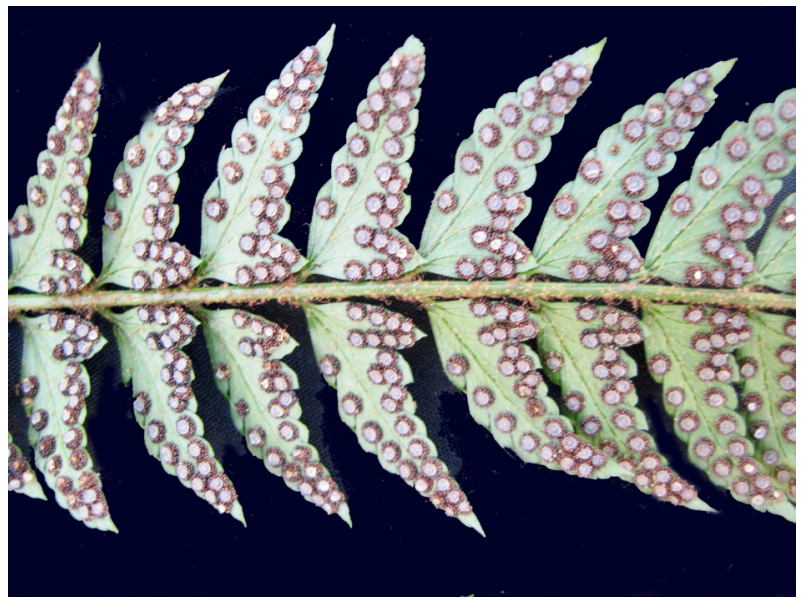

Image 7. Polystichum pseudotsus-simense. (C) Authors 


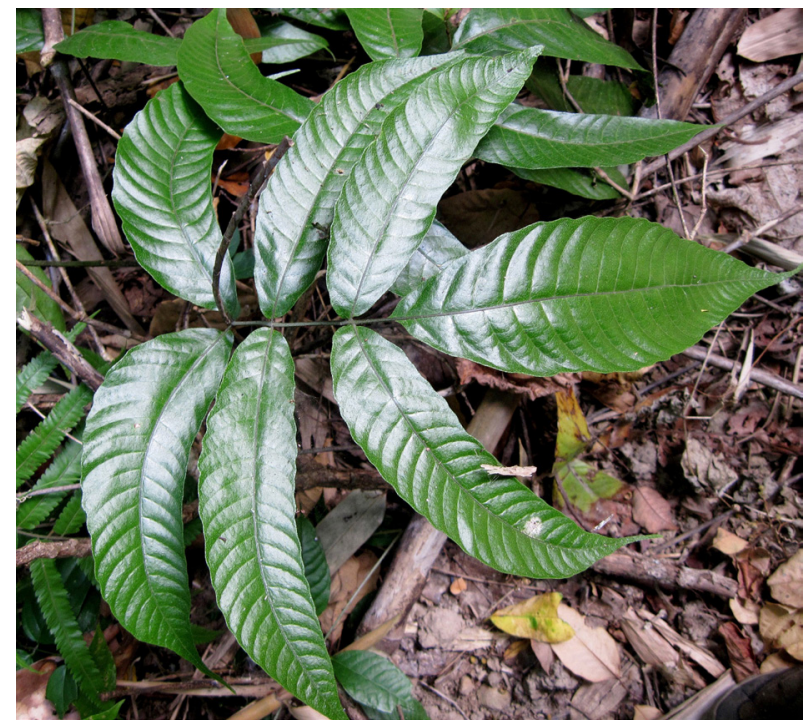

Image 8. Tectaria polymorpha. (c) Authors

forked; main vein 6-8 mm apart, curving upwards; crossveins arching, enclosing two irregular row of areoles with included veinlets. Sori indusiate round, rather small, in a single row on each side of each cross-vein, usually 4-6 in a row at the mid-pinna; indusia brown, thin, persistant, reniform.

Specimen examined: 124278, 30.xi.2011, terrestrial, TWS, coll. S.K. Singh \& H.A. Barbhuiya.

Distribution: Arunachal Pradesh (Singh \& Panigrahi 2005), Manipur (Malick \& Safui 1987), Meghalaya (Baishya \& Rao 1982), Mizoram (Benniamin 2012), Nagaland (Jamir \& Rao 1988) and in other parts of India and the world.

\section{Lomariopsidaceae}

32. Bolbitis heteroclita (C. Presl) Ching in Christenson, Index Fil., Suppl. 3, 48. 1934. Acrostichum heteroclitum C. Presl, Reliq. Haenk. 1(1): 15, t. 2, f. 2. 1825.

Rhizome long creeping, scaly; scales nearly black with narrow brown ferrugineous margin, linear, up to 5 by $1 \mathrm{~mm}$. Sterile fronds lamina simple or imparipinnate with one or two pairs of lateral pinnae; lateral pinnae oblong, cuneate and shortly stalked at base, caudate at apex, terminal pinna oblong, or often very long-tailed with narrow linear tails 50 . Fertile fronds lateral pinnae up to four pairs, oblong, apical pinna a little larger than lateral ones, veins reticulate; sporangia spread over the whole undersurface of pinnae.

Specimen examined: 124293, 30.xi.2011, lithophytic, TWS, coll. S.K. Singh \& H.A. Barbhuiya.

Distribution: Arunachal Pradesh (Singh \& Panigrahi 2005), Assam (Nayar \& Kaur 1964a), Meghalaya (Baishya
\& Rao 1982), Tripura (Vasudeva et al. 1990), Mizoram (Fischer 1938), Nagaland (Jamir \& Rao 1988) and in other parts of the world.

33. Bolbitis nodiflora (Bory) Fraser-Jenk., Syndr. Indian Pteridol. 65. 1997. Polybotrya nodiflora Bory, Bel. Voy. Bot. 2. 17. 1833.

Sterile fronds lamina oblong-lanceolate, pinnate, pinnae sub-opposite, gradually narrowing upwards, lowermost pinnae largest, pinnae almost unlobed, or very shallowly lobed. Fertile frond longer than the sterile one unipinnate, pinnae nodulose.

Specimen examined: 124396, 30.xi.2011, lithophytic, TWS, coll. S.K. Singh \& H.A. Barbhuiya.

Distribution: Arunachal Pradesh (Banniamin 2010), Meghalaya (Nayar \& Kaur 1964b as Egenolfia vivipara (Hook.) C. Chr.), Manipur, Nagaland (Chandra et al. 2008), Mizoram, Tripura (Deb 1981 as E. appendiculata (Willd.) J. Sm. var. vivipara Ham. ex Hook) and in other parts of the world.

34. Bolbitis sinensis (Baker) K. Iwats., Acta Phytotax. Geobot. 18: 49. 1959. Acrostichum sinense Baker, Bull. Misc. Inform. Kew 1906: 14. 1906.

Sterile fronds stipe scaly at base, lamina narrowly subtriangular, the apex attenuately caudate, viviparous subapically. Fertile fronds about the same length or taller than the sterile ones, lamina narrower, lateral pinnae oblong, gradually narrowing from base to apex, subentire or very slightly waved at margin, sporangia dispersed on the undersurface, naked.

Specimen examined: 124285, 30.xi.2011, lithophytic, TWS, coll. S.K. Singh \& H.A. Barbhuiya.

Distribution: Arunachal Pradesh (Singh \& Panigrahi 2005), Assam (Nath \& Bhattacharaya 2002), Meghalaya (Baishya \& Rao 1982 as Bolbitis bipinnatifida (J. Sm.) K. Iwats.), Mizoram (Deb \& Dutta 1987 as Egenolfia bipinnatifida J. Sm.; Benniamin 2012), Nagaland (Jamir \& Rao 1988 as Bolbitis bipinnatifida), Tripura (Deb 1981 as Egenolfia bipinnatifida) and in other parts of the world.

35. Bolbitis virens (Wall. ex Hook. \& Grev.) Schott, Gen. Fil. t. 13. 1834. Acrostichum virens Wall. ex Hook. \& Grev., Icon. Filic. 2(11): t. 221. 1831.

Sterile fronds pinnate ca. $60 \mathrm{~cm}$ long; stipe stramineous, lamina oblong-lanceolate, acute at apex; lateral pinnae 12-20 pairs, alternate, linear-lanceolate, usually widest at middle, base broadly attenuate or cuneate, margin slightly serrate-crenate or entire, apex acuminate, lowermost two pinnae with short stalk; terminal segment similar to central pinnae; veins 
forming a costal areole and several to many equally large distal ones, four veinlets arising from either side of lateral veins. Fertile fronds almost equal in height of sterile ones; pinnae usually 9-10 pairs, linear up to $6 \mathrm{~cm}$ long. Sporangia inserted usually throughout abaxial surface, arrangement various.

Specimen examined: 124297, 30.xi.2011, terrestrial, TWS, coll. S.K. Singh \& H.A. Barbhuiya.

Distribution: Assam (Barbhuiya 2013), Meghalaya (Baishya \& Rao 1982) and in other parts of India and the world. This is reported here for the first time from Mizoram.

\section{Blechnaceae}

\section{Blechnum orientale L., Sp. PI. 1077. 1753.}

Rhizome thick, ascending or suberect, densely covered with scales. Stipe with small, alternate, knob like aerophores. Laminae long, lateral pinnae many in number, ascending, linear, gradually narrowing towards long-tailed apex, round or subtruncate at sessile base. Sori narrow, long-continuous along costa; indusia narrow.

Specimen examined: 124283, 30.xi.2011, terrestrial, TWS, coll. S.K. Singh \& H.A. Barbhuiya.

Distribution: Arunachal Pradesh (Singh \& Panigrahi 2005), Assam (Bir et al. 1990), Manipur (Malick \& Safui 1987), Meghalaya (Baishya \& Rao 1982), Mizoram (Gage 1901; Benniamin, 2012), Nagaland (Jamir \& Rao 1988), Tripura (Deb 1981) and in other parts of India and the world.

\section{REFERENCES}

Alston, A.H.G. (1945). An enumeration of the Indian species of Selaginella. Proceedings of the National Institute of Sciences of India 11(3): 211-235.

Baishya, A.K. \& R.R. Rao (1982). Ferns and Ferns-allies of Meghalaya State, India. Scientific Publishers, Jodhpur, India, 192pp.

Barbhuiya, H.A. (2013). Study and assessment of threatened and endemic vascular plants of southern Assam. PhD Thesis submitted to Assam University, Silchar, Assam, India.

Beddome, R.H. (1863). The Ferns of Southern India. Gantz Bros. Madras, 1-271pp.

Beddome, R.H. (1892). Handbook to the Ferns of British India Ceylon and the Malay Peninsula. Thacker Spink and Co., Calcutta, India, $110 \mathrm{pp}$

Benniamin, A. (2010). Fern and fern-allies of Itanagar Wildlife Sanctuary. Indian Journal of Forestry 33(2): 199-205.

Benniamin, A. (2011). Studies on the genus Dryopteris Adans (Pteridophyta-Aspidiaceae Sensu Lato) from Mizoram (North East India). Indian Journal of Forestry 34(3): 345-352.

Benniamin, A. (2012). The diversity of ferns and fern allies of Murlen National Park, Mizoram, North East India. Indian Fern Journal 29: 1-12.

Bir, S.S. (1998). Monograph of Aspleniod ferns of India: Systematics and taxonomy I. Subgenera Neottopteris, Asplenidictyum, Phyllitis, Ceterachopsis and Ceterach. Indian Fern Journal 15: 205-205.

Bir, S. S., S.M. Vasudeva \& P. Kachroo (1989). Pteridophytic flora of north-eastern India - I (Families: Huperziaceae-Sinopteridaceae). Indian Fern Journal 6: 30-55.

Bir, S. S., S.M. Vasudeva \& P. Kachroo (1990). Pteridophytic flora of north-eastern India - IV (Families: Davalliaceae-Salviniaceae). Indian Fern Journal 7: 219-226.

Bir, S.S., S.M. Vasudeva, P. Kachroo \& Y. Singh (1991). Pteridophytic flora of north-eastern India - V ecological, distributional and phytogeographic account. Indian Fern Journal 8: 98-139.

Blatter, E. \& J.F. d'Almedia (1922). The Ferns of Bombay. D.B. Taraporevala Sons and Co., Bombay, India, 228pp.

Borthakur, S.K., P. Deka \& K.K. Nath (2001). Illustrated Manual of Fern of Assam. Bishen Singh Mahandra Pal Singh, Dehradun, India, 468pp.

Brummitt, R.K. \& C.E. Powell (1992). Authors of Plant Names. Royal Botanical Gardens. Kew, London, UK, 732pp.

Chandra, P. \& S. Chandra (1983). Contribution to the ferns of Mizoram. Journal of Bombay Natural History Society 80: 461-465.

Chandra, S., C.R. Fraser-Jenkins, A. Kumari \& A. Srivastava (2008). A summary of the status of threatened Pteridophytes of India. Taiwania 53(2): 170-209.

Clarke, C.B. (1880). A review of the ferns of northern India (Part I.). Transactions of the Linnean Society of London. Botany 1(7): 425611.

Clarke, C.B. (1890). On the plants of Kohima and Muneypore. Journal of the Linnean Society, Botany 25: 1-107.

Das, N.C. (1992). Taxonomic studies in Thelypteriod ferns of Tripura state, Eastern India. Indian Fern Journal 9: 233-239.

Deb, D.B. (1981). The Flora of Tripura State Vol. 1. Today and Tomorrow's Printers and Publishers, New Delhi, India, 31-64pp.

Deb, D.B. \& R.M. Dutta (1987). A contribution to the flora of Mizoram. Journal of Economic and Taxonomic Botany 10(1): 21-61.

Dixit, R.D. (1984). A census of the Indian pteridophytes. Botanical Survey of India, Howrah, India. $177 \mathrm{pp}$.

Dixit, R.D. (1998). Taxonomic studies on the family Cyatheaceae (Tree ferns) in India. Indian Fern Journal 15: 29-43.

Fischer, C.E.C. (1938). The flora of Lushai hills. Records of the Botanical Survey of India 12: 75-161.

Fraser-Jenkins, C.R. (1997). New Species Syndrome in Indian Pteridology and Ferns of Nepal. International Book Distributors, Dehra Dun, 403pp.

Fraser-Jenkins, C.R. (2008). Taxonomic Revision of Three Hundred Indian Subcontinental Pteridophytes with a Revised Census-List, a new picture of fern-taxonomy in the Indian subcontinent. Bishen Singh Mahendra Pal Singh, Dehra Dun, 685pp.

Fraser-Jenkins, C.R. (2009). A brief comparison of modern pteridophyte classifications (families and genera in india). Indian Fern Journal 26: 107-126

Fraser-Jenkins, C.R. (2012). Rare and threatened Pteridophytes of Asia 2. Endangered species of India - the higher IUCN categories. Bulletin of the National Museum of Nature and Science. Series $B$ 38(4): 1-29.

Gage, A.T. (1901). A botanical tour in the South Lushai Hills. Records of the Botanical Survey of India 1: 331-369.

Ghosh, S.R., B. Ghosh, A. Biswas \& R.K. Ghosh (2004). The Pteridophytic Flora of Eastern India Vol. - 1. Botanical Survey of India, Kolkata, India, 591pp.

Holttum, R.E. (1979). Sphaerostephanos (Thelypteridaceae) in Asia, excluding Malesia. Kew Bulletin 34(2): 221-232

Hooker, W.J. (1862). Species Filicum Vol. 4. William Pamplin, London, UK, 292pp.

Jamir, N.S. \& R.R. Rao (1988). Ferns of Nagaland. Bishen Singh Mahandra Pal Singh, Dehradun, India, 426pp.

Kachroo, P., S.S. Bir \& S.M. Vasudeva (1989). Pteridophytic flora of north-eastern India - II (Families: CryptogrammaceaeThelypteridaceae). Indian Fern Journal 6: 78-99.

Kholia, B.S. (2010). Fern and Fern-allies of Sikkim, A Pictorial Handbook Part - I. Sikkim State Biodiversity Board, Sikkim and Botanical Survey of India, Kolkata, India, 207pp.

Kumar, R., H.A. Barbhuiya, A. Benniamin \& S.K. Singh (2012). Cyathea 
brunoniana - a less known Pteridophyte from Kolasib, Mizoram. Keanean Journal of Science 1: 16-19.

Mehra, P.N. (1932). Ceratopteris calomelanos in Sikkim. Journal of the Indian Botanical Society 11: 340-341.

Mehra, P.N. (1939). Ferns of Mussoorie. Punjab University Botanical Publications, Lahore, Pakistan, 1-29pp.

Malick, K.C. \& B. Safui (1987). Additions to the flora of Manipur State. Journal of Economic and Taxonomic Botany 10(1): 1-19.

Nath, A. \& M.K. Bhattacharaya (2002). Some new records and little known species of pteridophytes from Barak Valley, Assam, India. Indian Fern Journal 19: 97-106.

Nayar, B.K. \& S. Kaur (1964a). Ferns of India - XI, Bolbitis. Bulletin of the National. Botanic. Garden, Lucknow 88: 1-74.

Nayar, B.K. \& S. Kaur (1964b). Ferns of India - XIII, Egenolfia. Bulletin of the National. Botanic. Garden, Lucknow 100: 1-38.

Panigrahi, G. (1975). The Genus Pityrogramma (Hemionitidaceae) in Asia. Kew Bulletin 30(4): 657-667

Shukla, U. \& A.K. Baishya (1980). A contribution to the flora of Manipur. Journal of the Bombay Natural History Society 76: 224-230.
Singh, S. \& G. Panigrahi (1984). Systematics of genus Lygodium Sw. (Lygodiaceae) in India. Proceedings of the Indian Academy of Sciences. Plant Sciences 93: 119-133.

Singh, S. \& G. Panigrahi (2005). Ferns and Fern Allies of Arunacha Pradesh, Vol. 1 \& 2. Bishen Singh Mahandra Pal Singh, Dehradun, India, 881pp.

Vasudeva, S.M., S.S. Bir \& P. Kachroo (1990). Pteridophytic flora of north-eastern India - III (Families: Aspleniaceae-Oleandraceae). Indian Fern Journal 7: 66-85.

Verma, S.C. (1966). A note on Pityrogramma calomelanos in India. Bulletin of the Botanical Survey of India 8(1): 99-100.

Verma, S.C. (1962 '1961'). Taxonomic status of Adiantum lunulatun Burm. Nova Hedwigia 3(4): 463-468.

Verma, S.C. \& C.R. Fraser-Jenkins (2008). Adiantum philippense L., the correct name for Adiantum lunulatum Burm. f. and its subspecies, pp. 65-92. In: Verma, S.C., S.P. Khullar \& H. Cheema (eds.). Perspectives in Pteridophytes. Bishen Singh Mahendra Pal Singh, Dehradun, India.
Appendix 1. Herbarium images

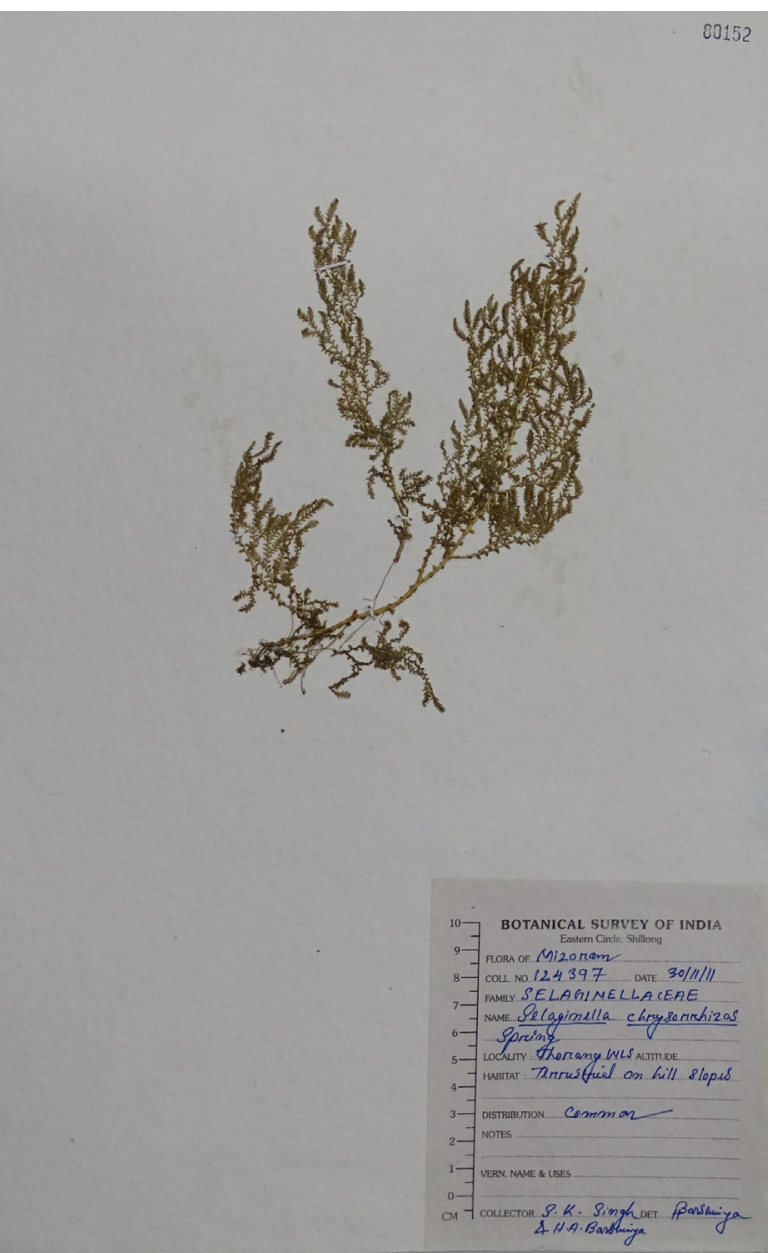

Image 9. Selaginella chrysorrhizos

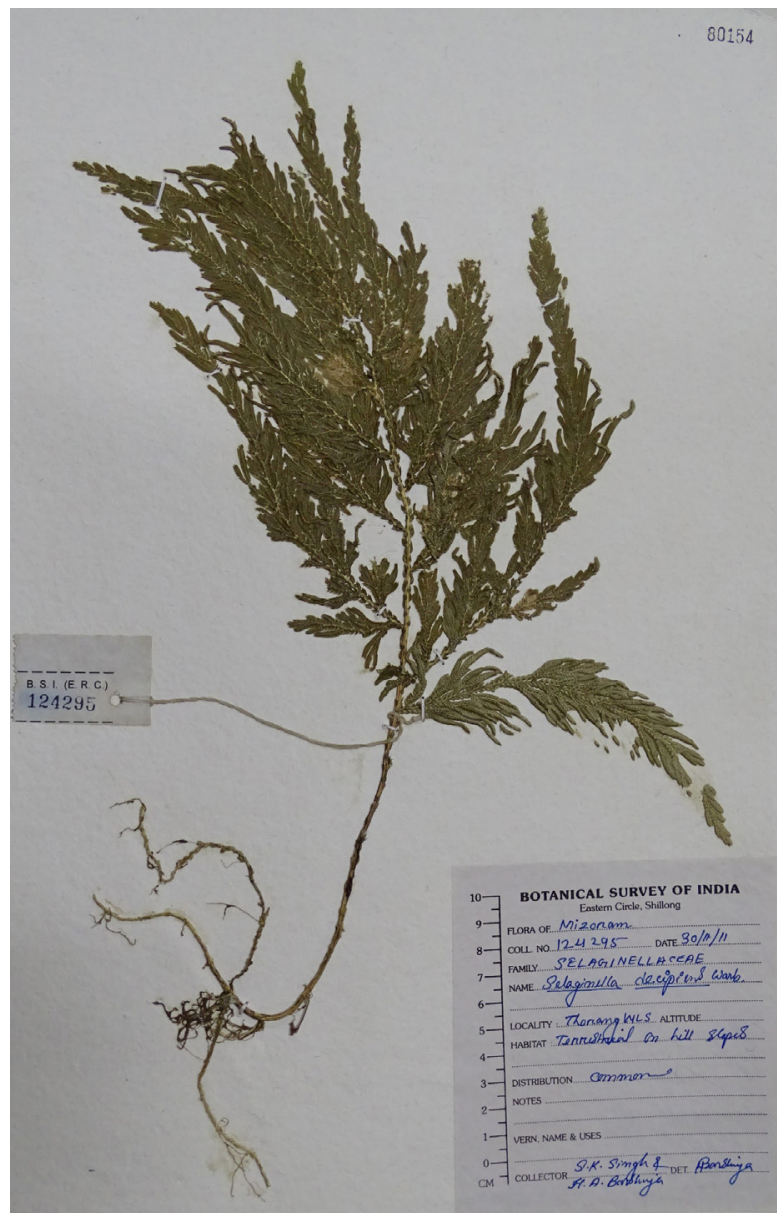

Image 10. Selaginella decipiens 


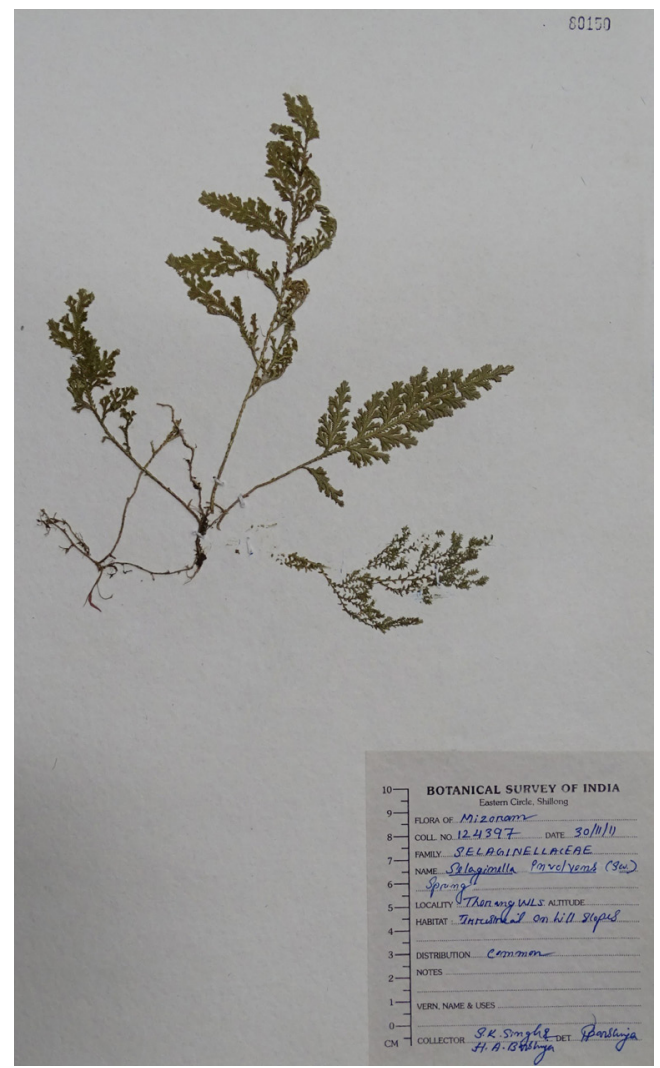

Image 11. Selaginella involvens

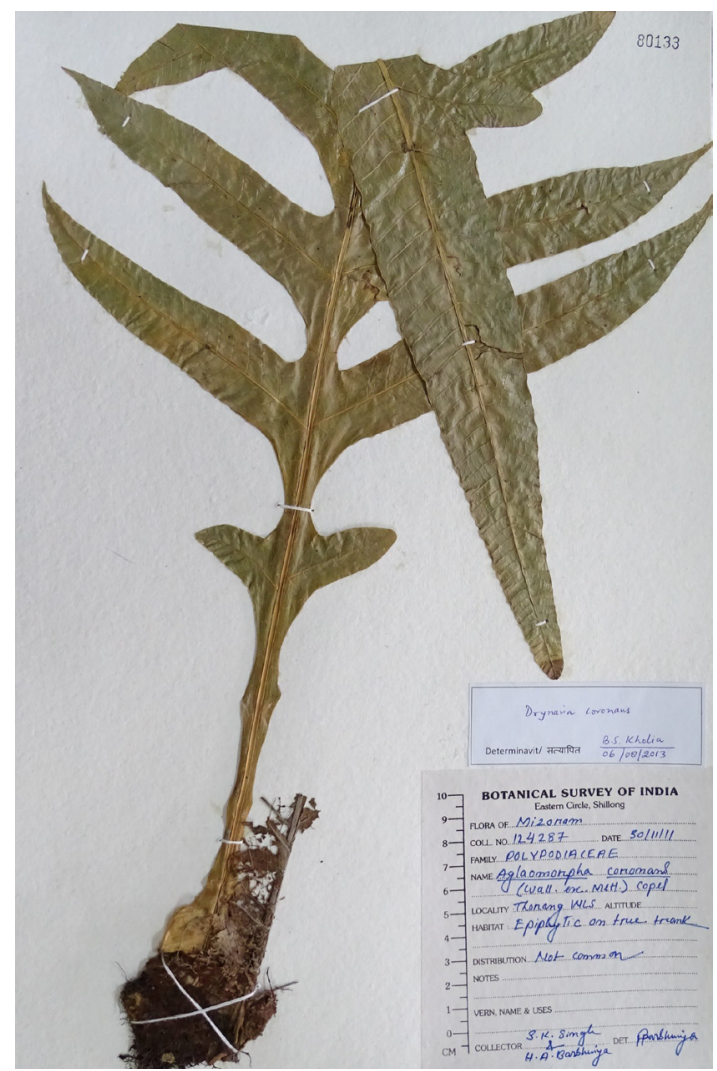

Image 13. Drynaria coronans

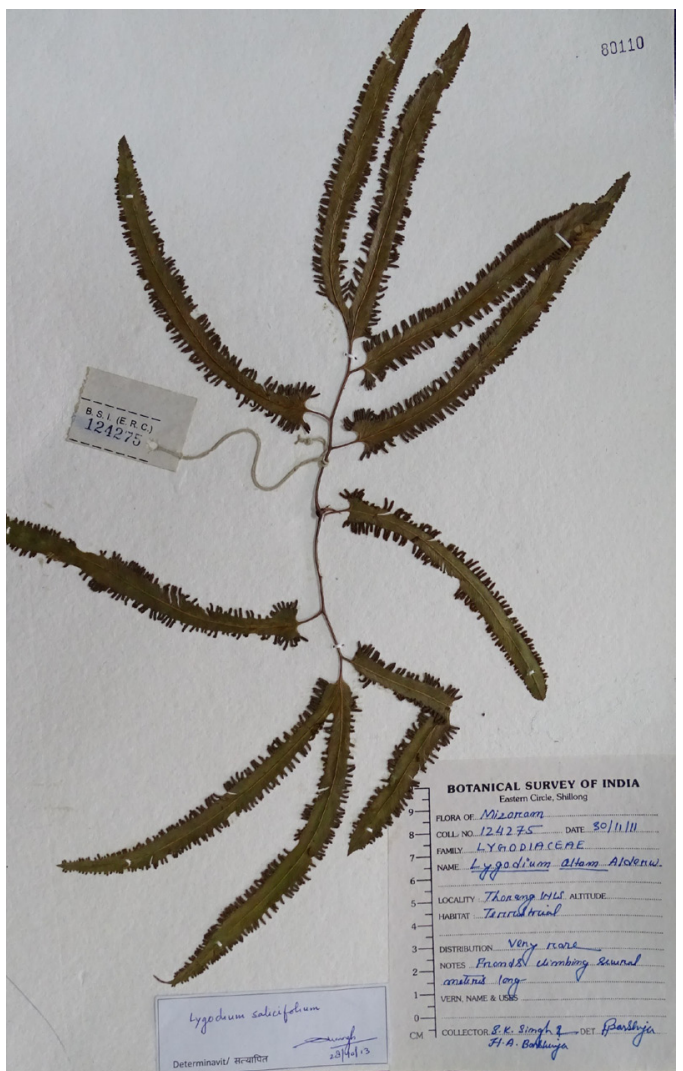

Image 12. Lygodium salicifolium

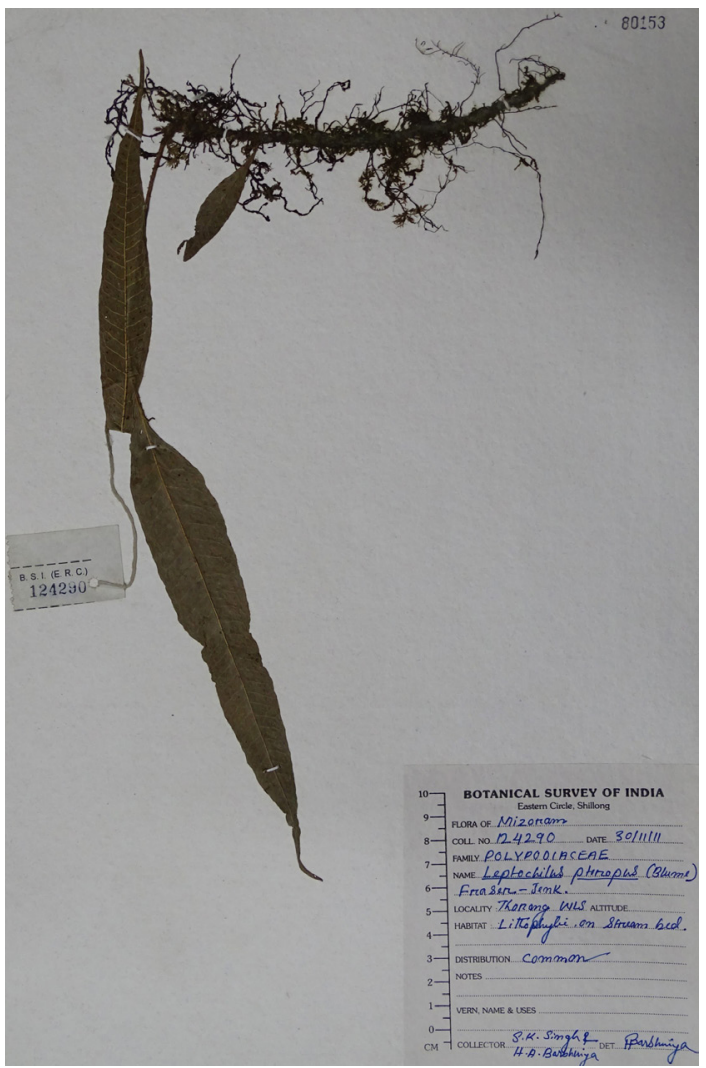

Image 14. Leptochilus pteropus 


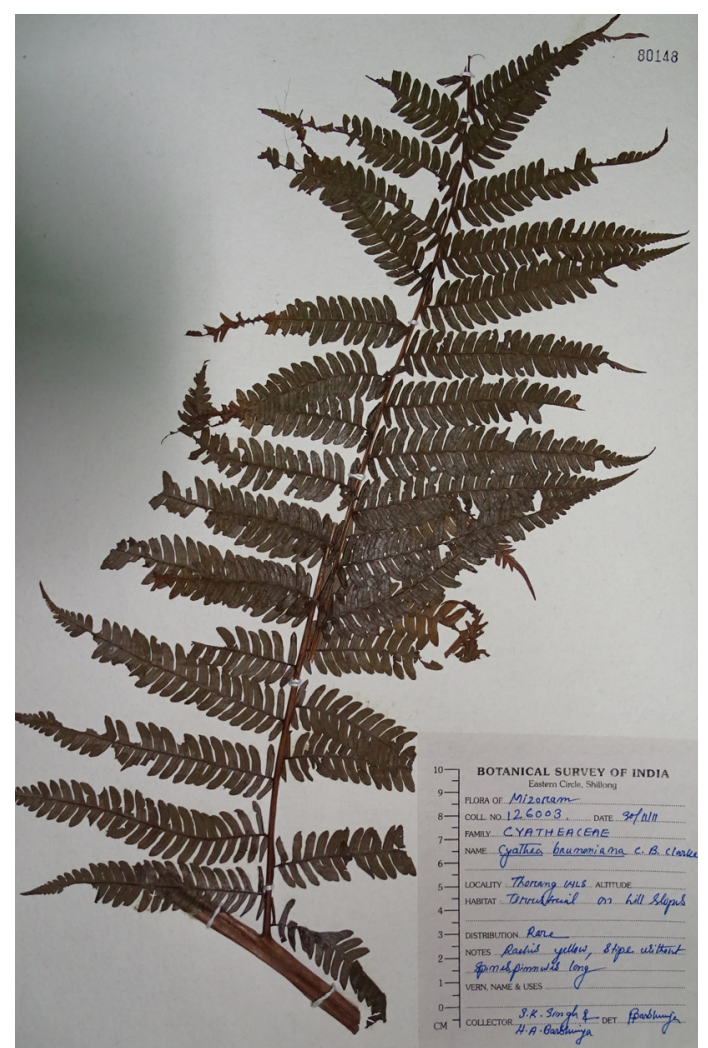

Image 15. Cyathea brunoniana

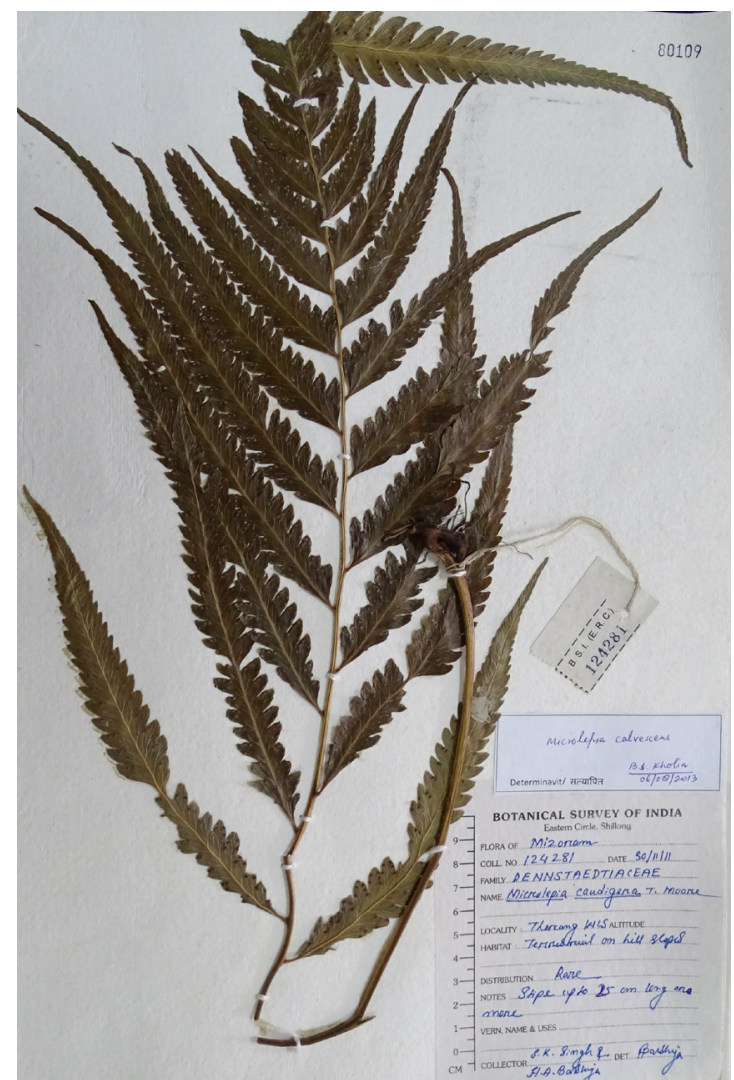

Image 17. Microlepia calvescens

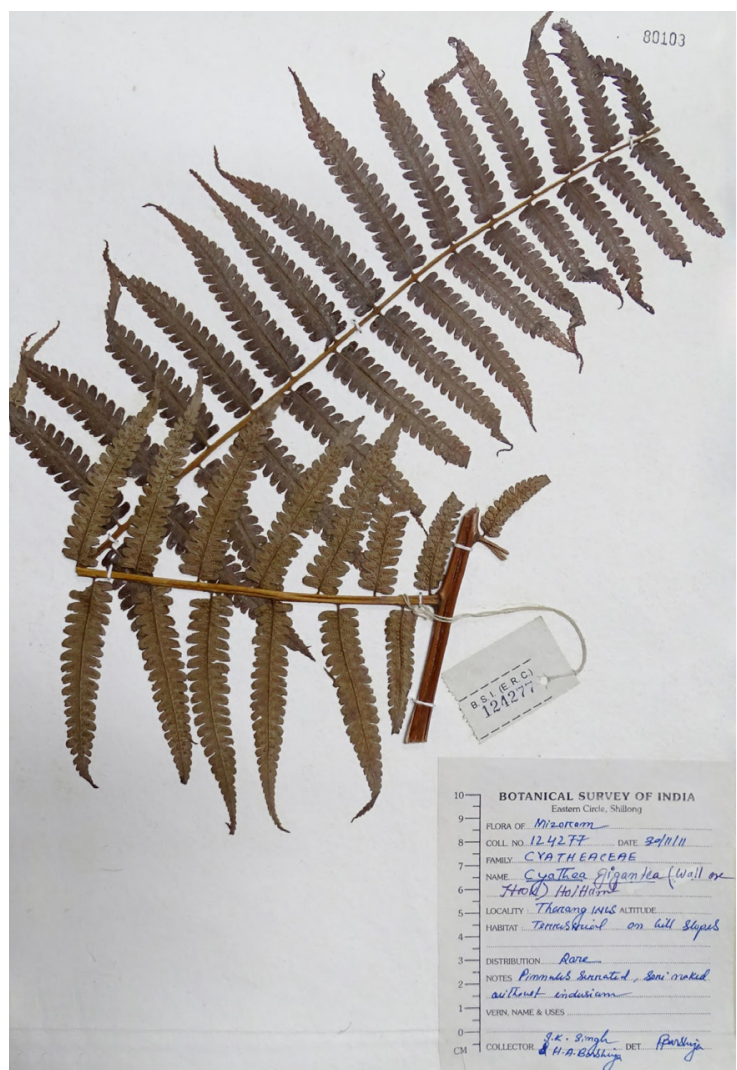

Image 16. Cyathea gigantea

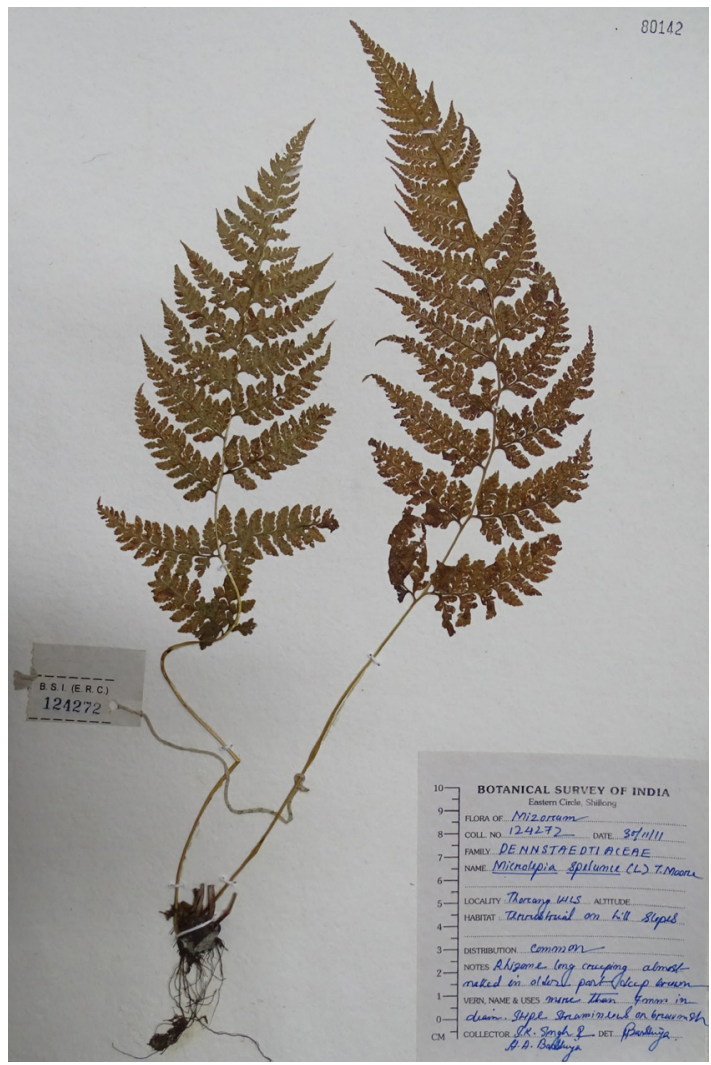

Image 18. Microlepia speluncae 


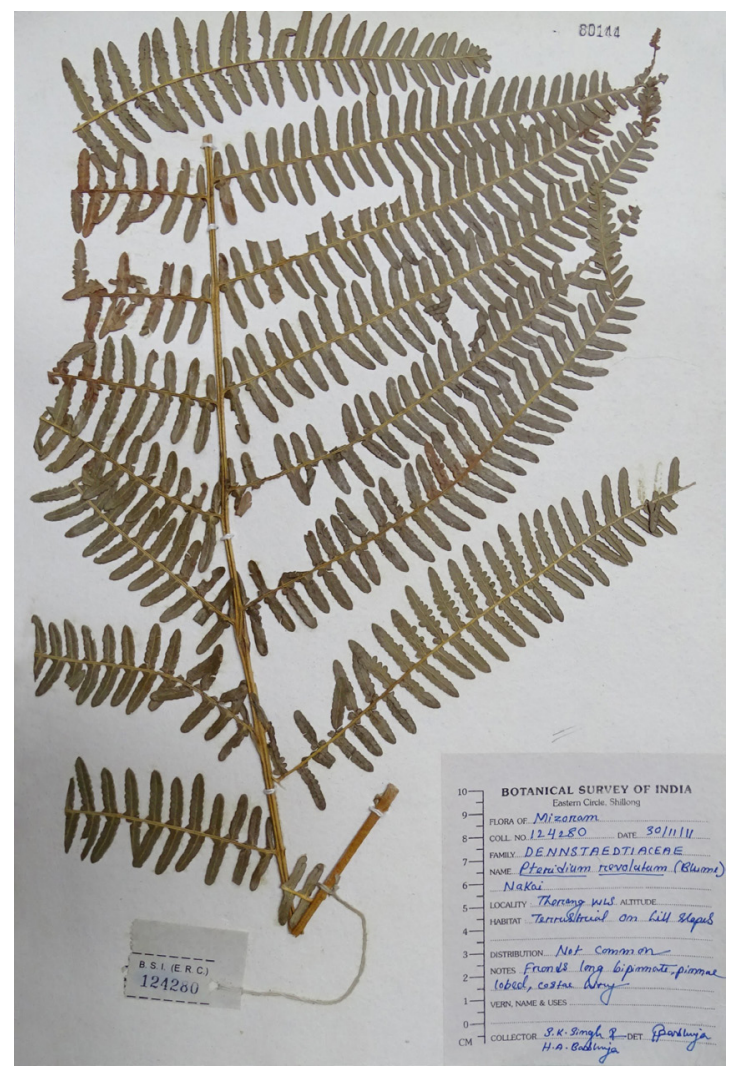

Image 19. Pteridium revolutum

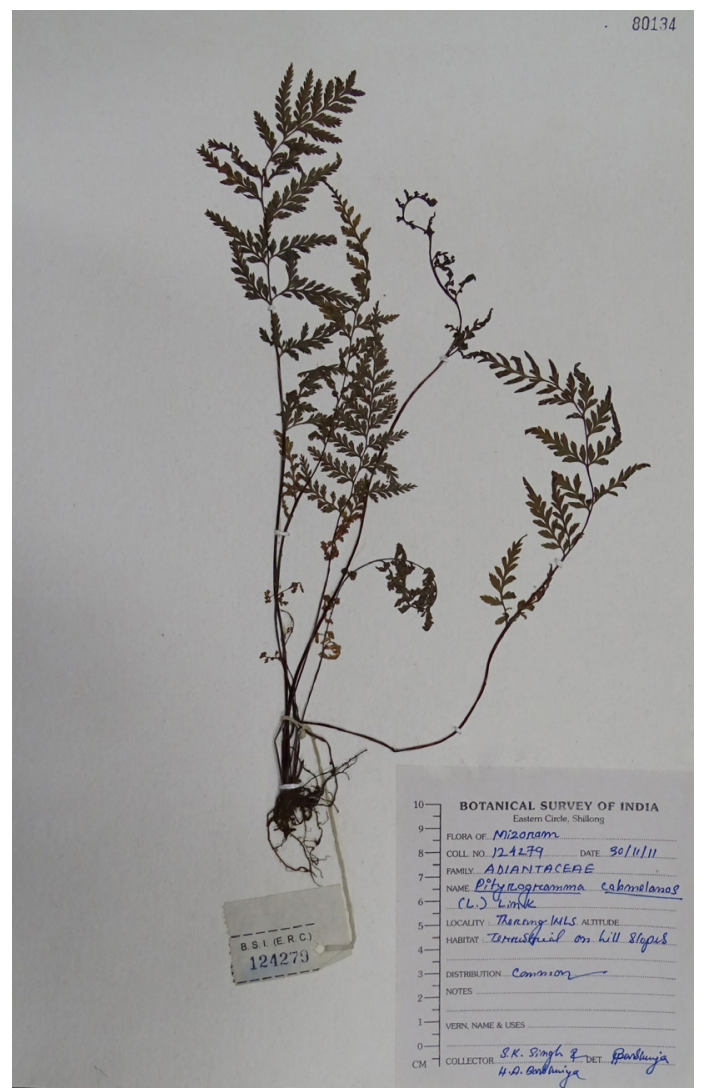

Image 21. Pityrogramma calomelanos

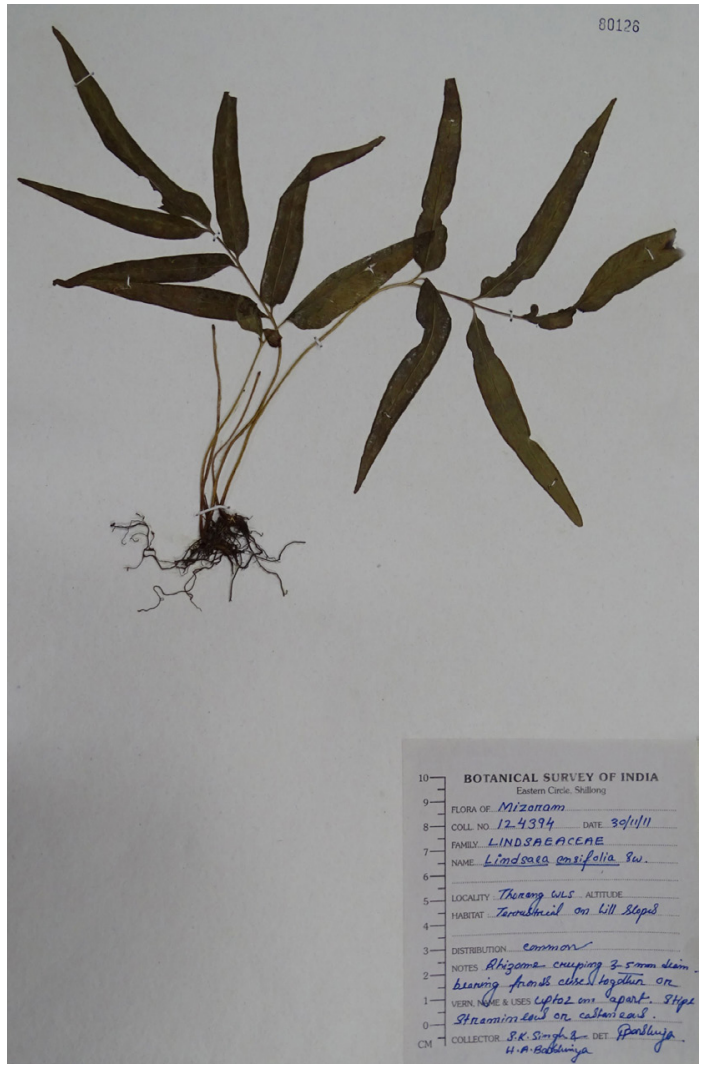

Image 20. Lindsaea ensifolia

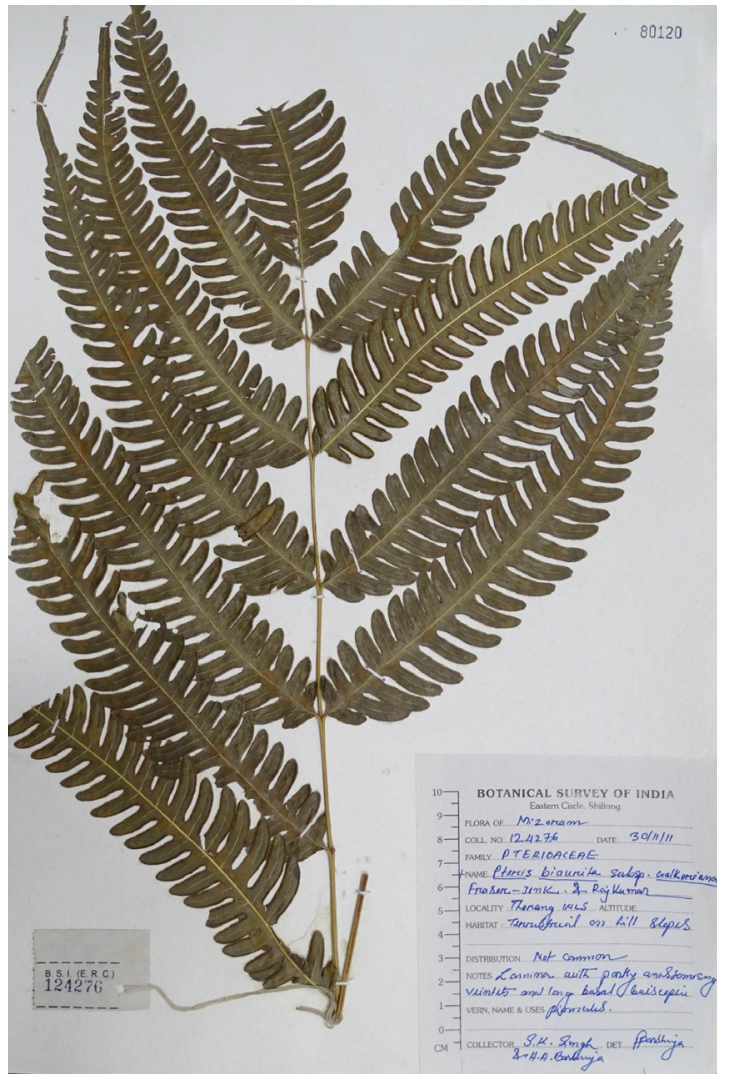

Image 22. Pteris biaurita L. subsp. walkeriana 


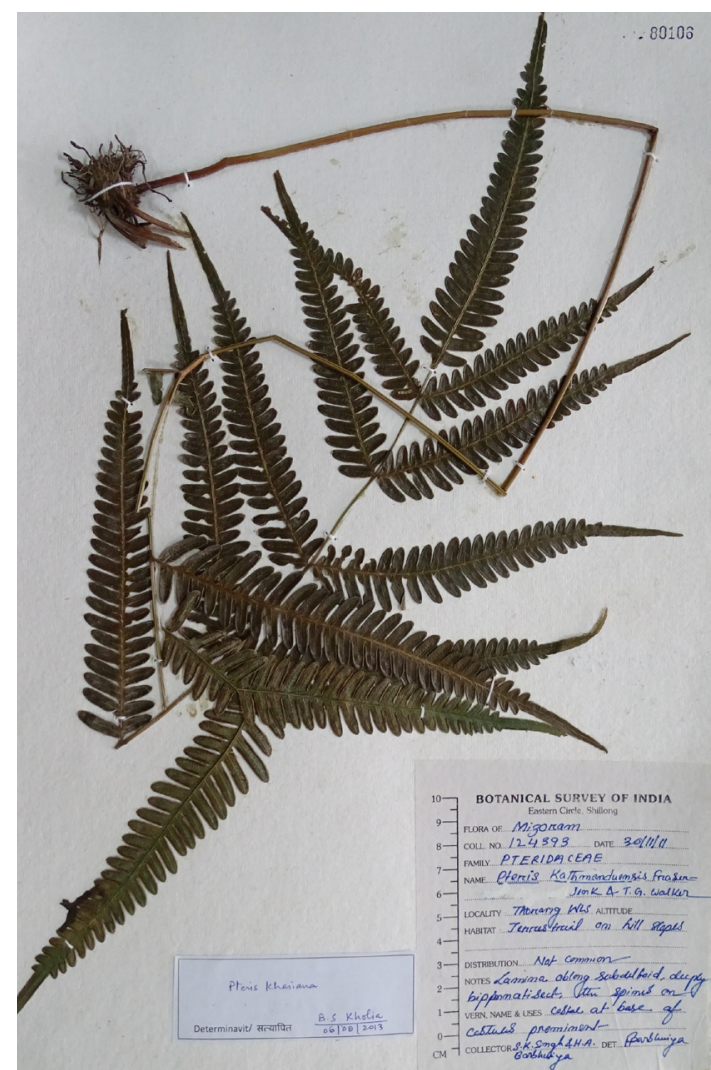

Image 23. Pteris khasiana

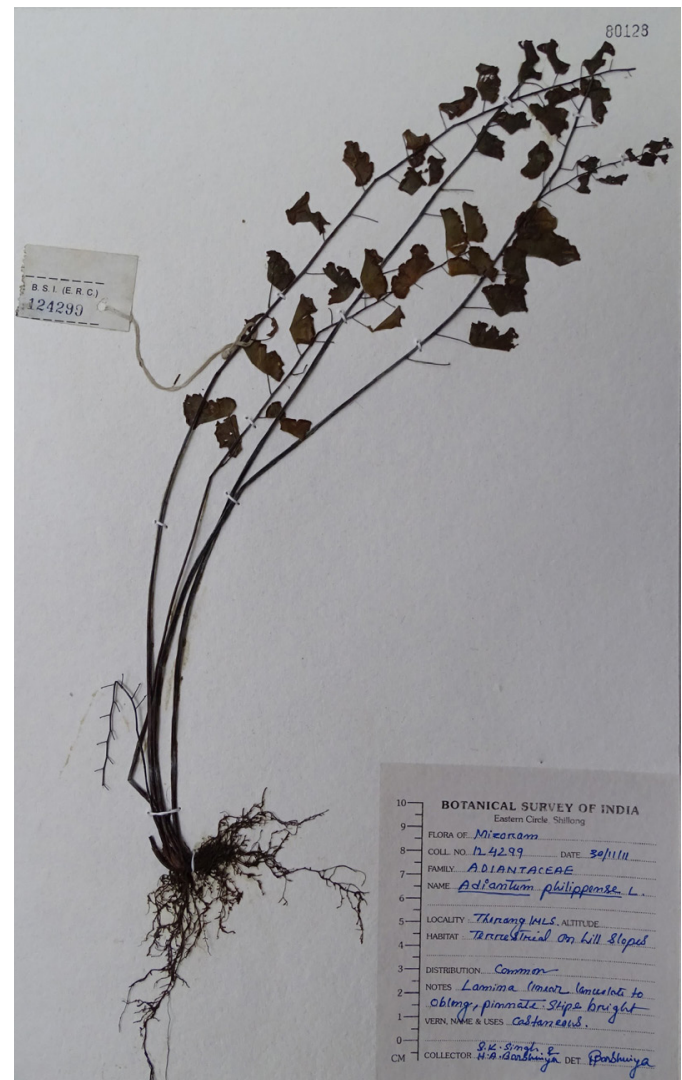

Image 25. Adiantum philippense

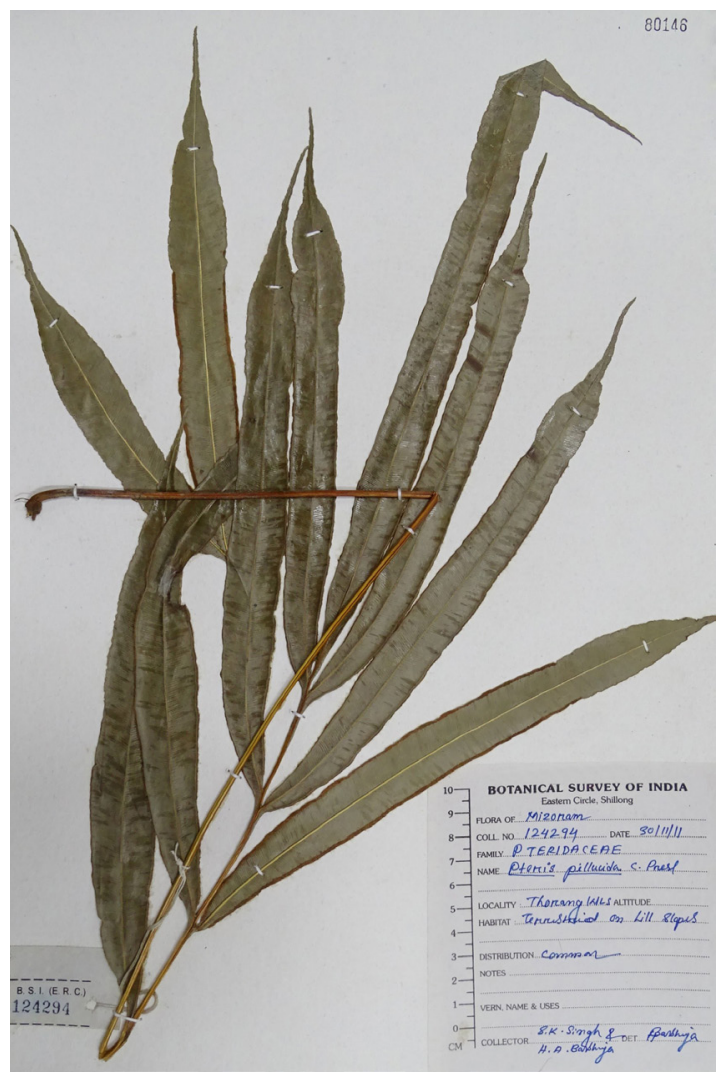

Image 24. Pteris pellucida

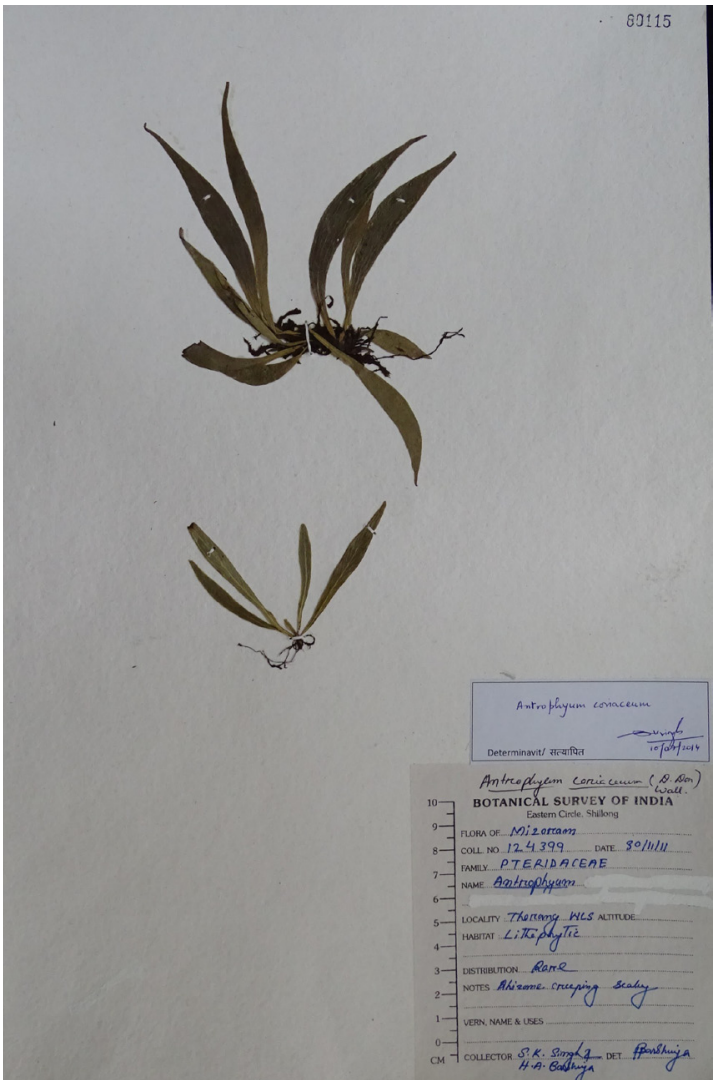

Image 26. Antrophyum coriaceum 


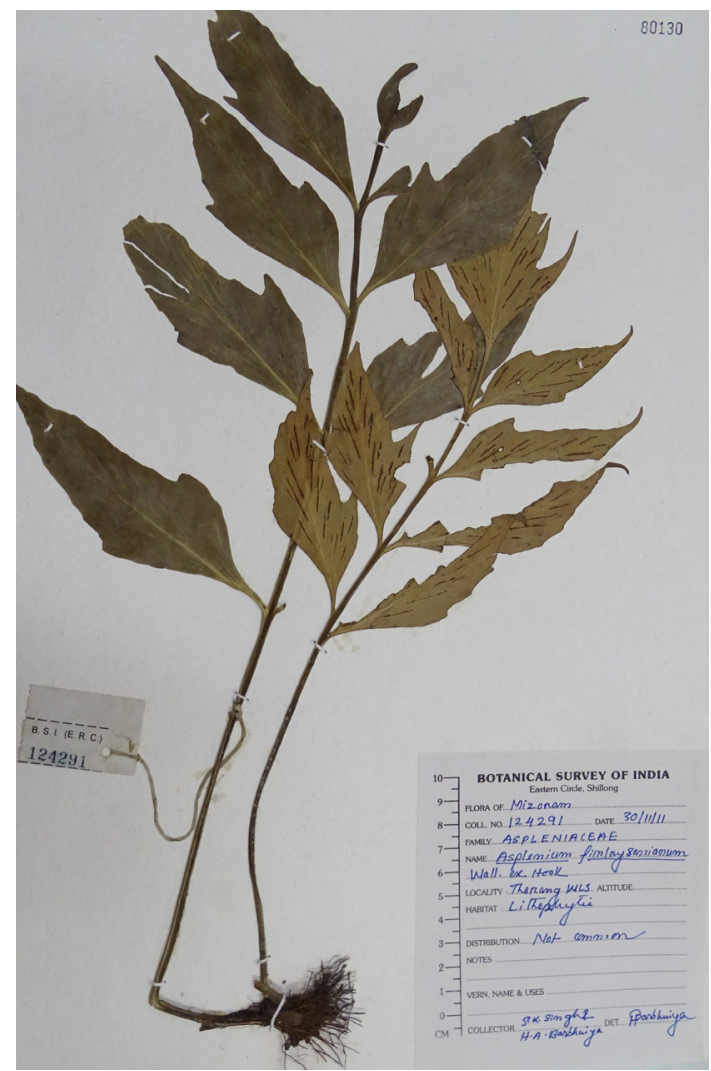

Image 27. Asplenium finlaysonianum

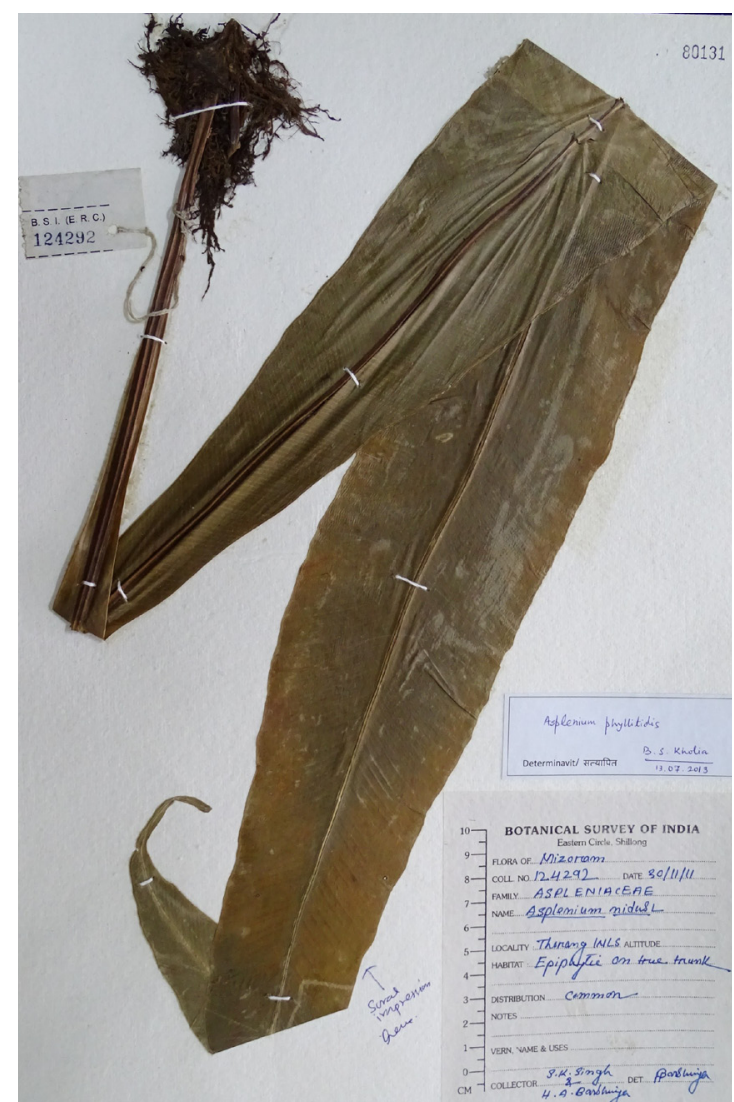

Image 29. Asplenium phyllitidis

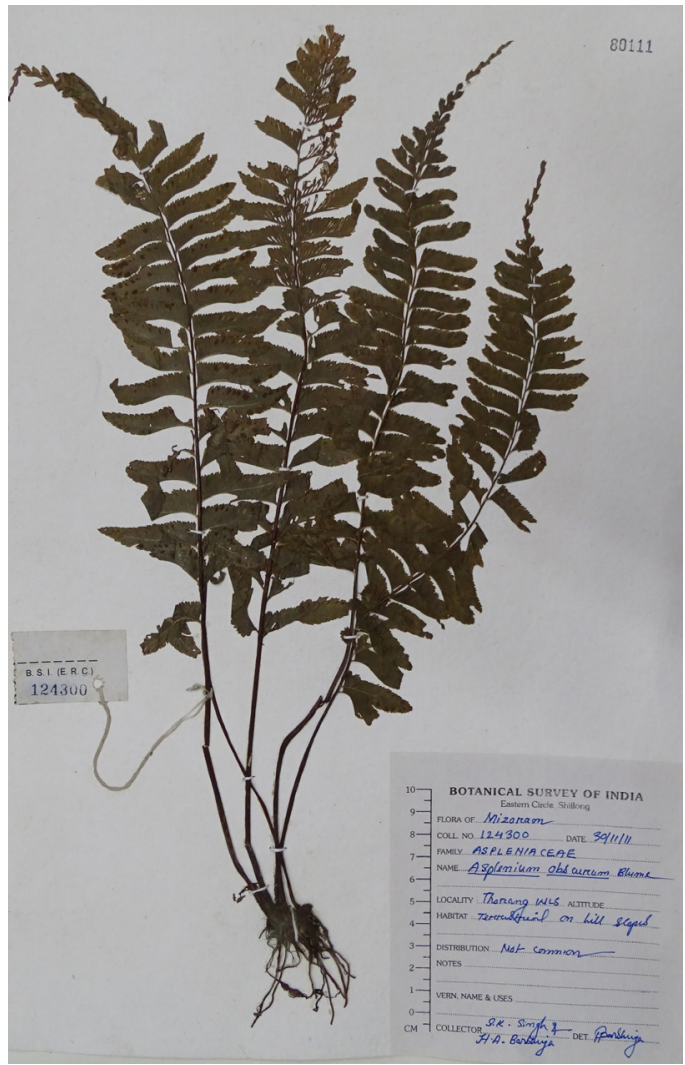

Image 28. Asplenium obscurum

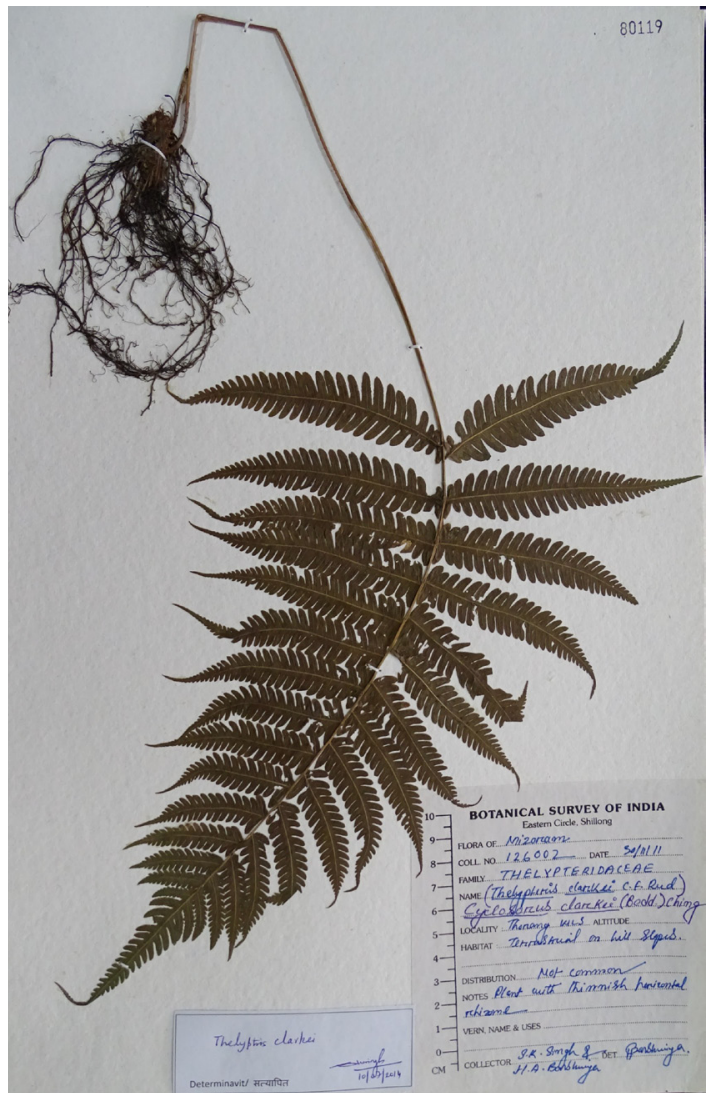

Image 30. Thelypteris clarkei 


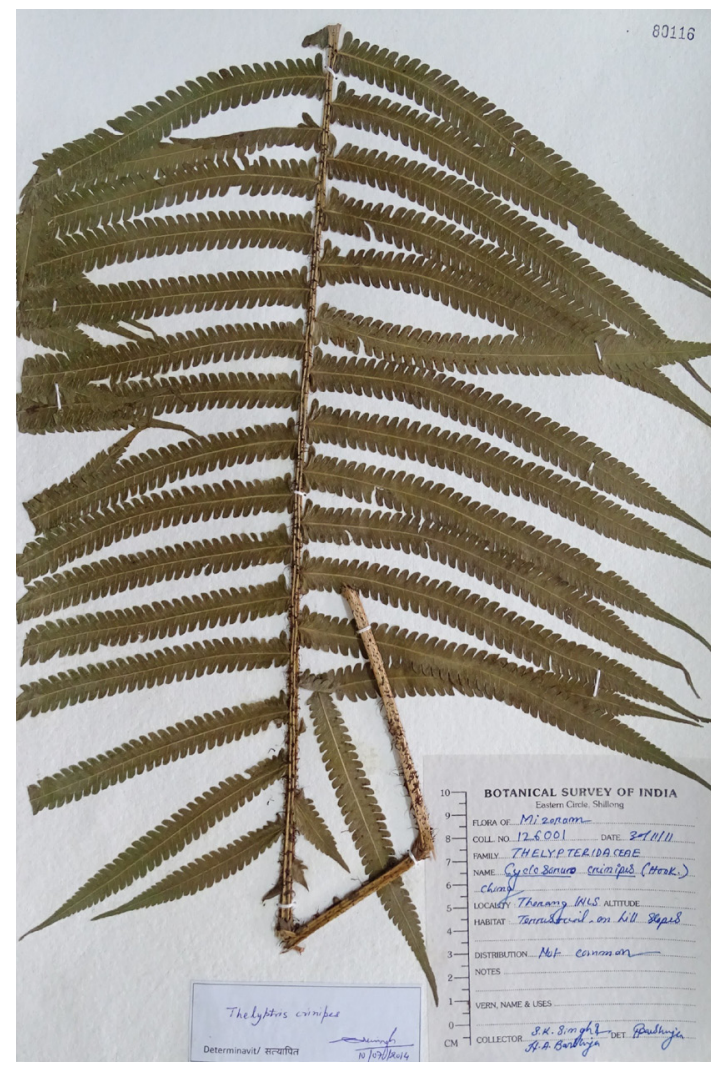

Image 31. Thelypteris crinipes

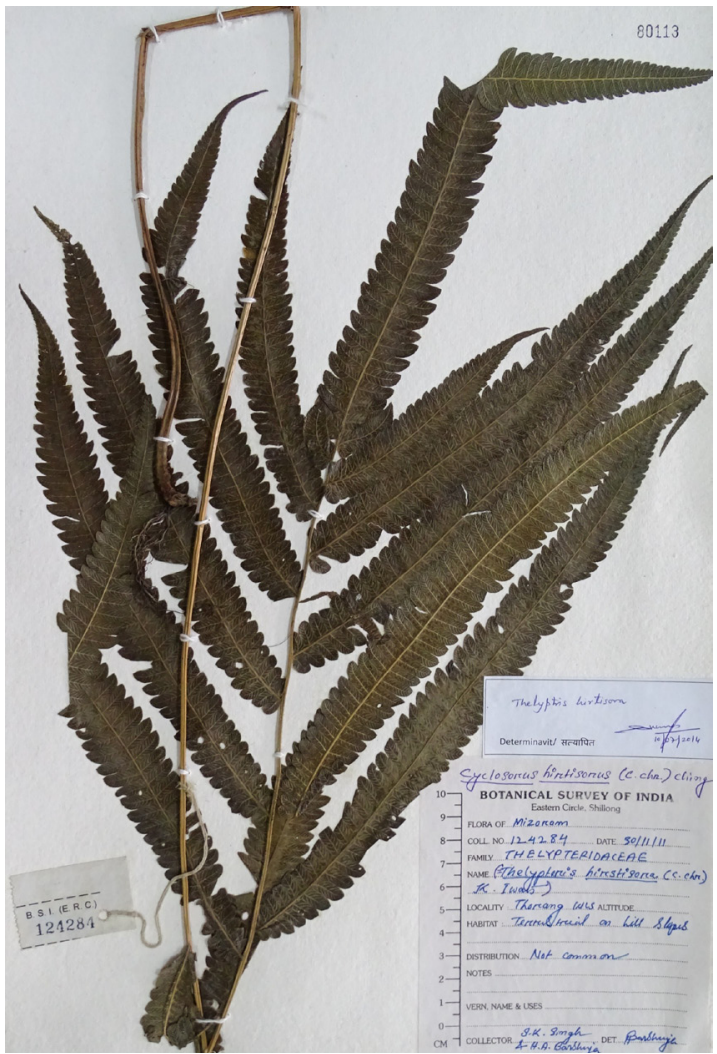

Image 33. Thelypteris hirtisora

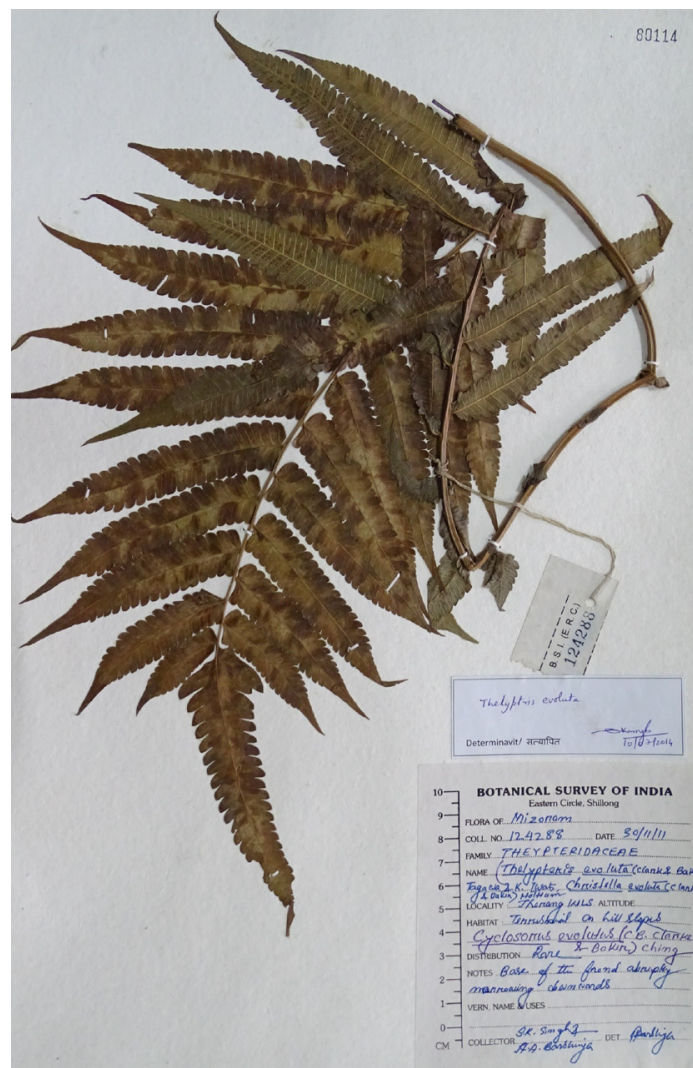

Image 32. Thelypteris evoluta

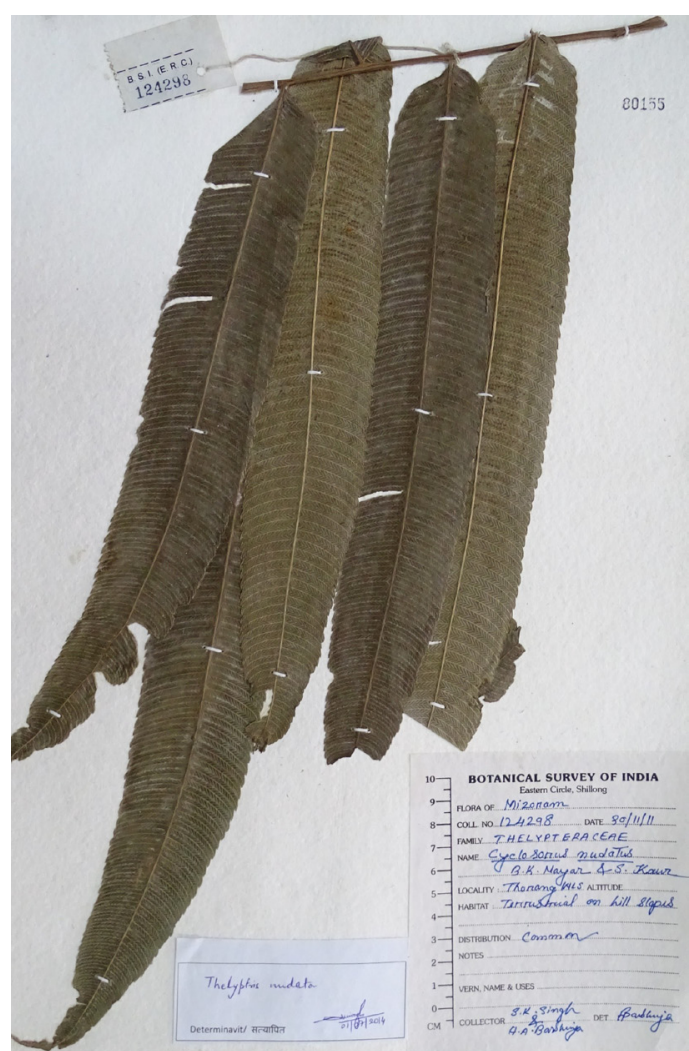

Image 34. Thelypteris nudata 


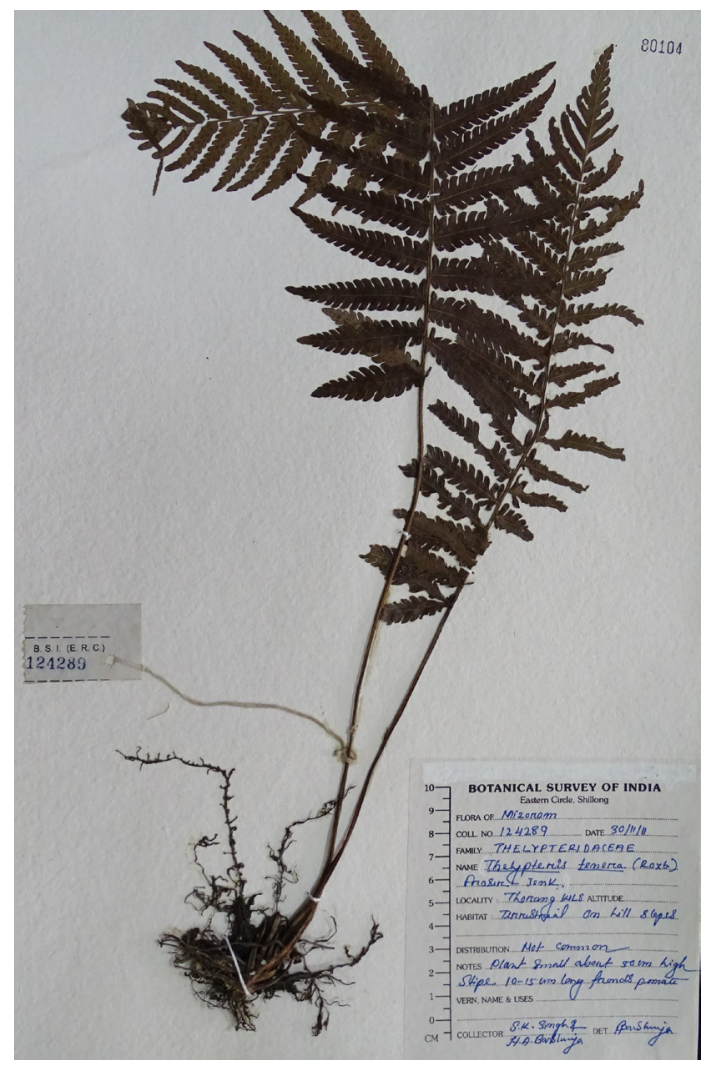

Image 35. Thelypteris tenera

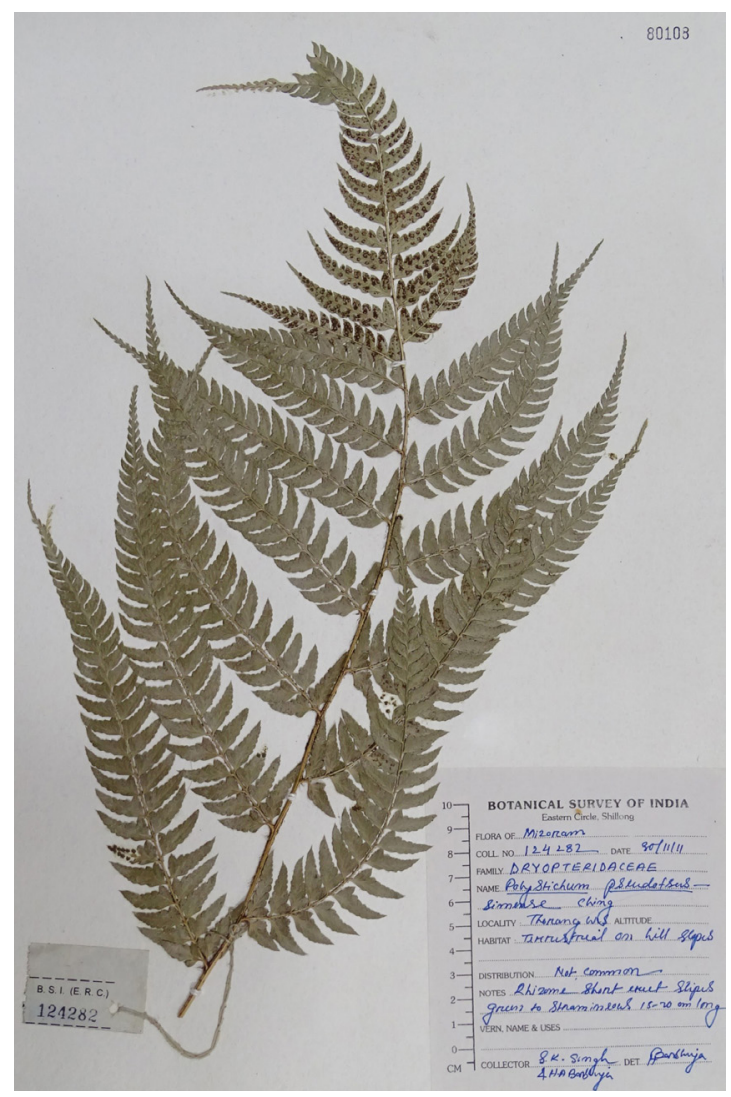

Image 37. Polystichum pseudotsus-simense

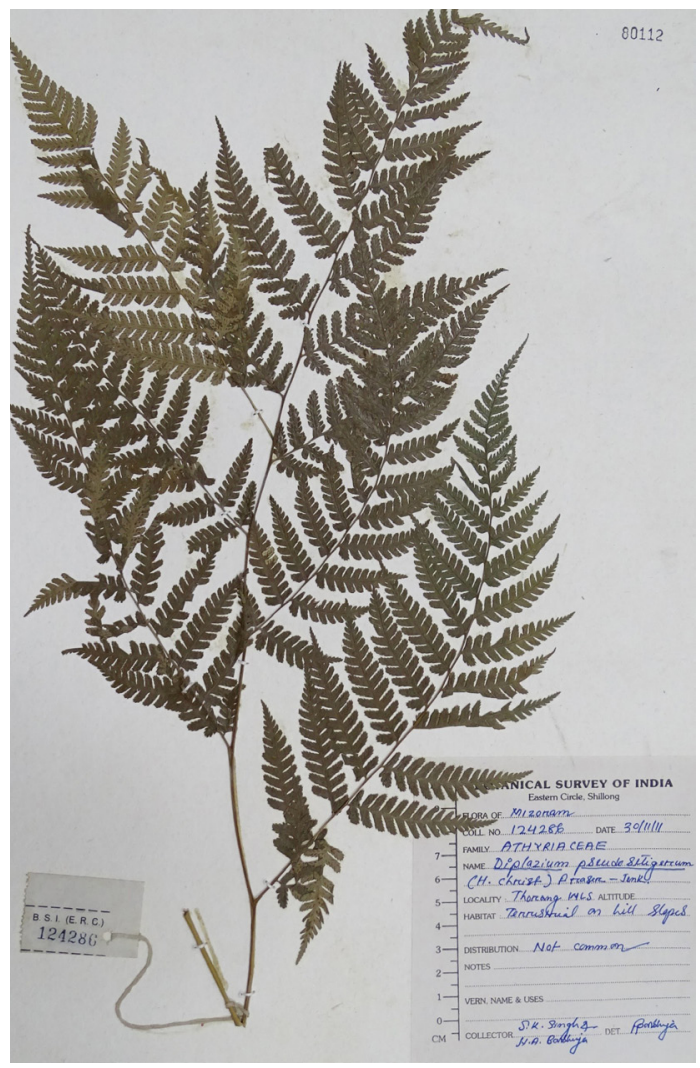

Image 36. Diplazium pseudosetigerum

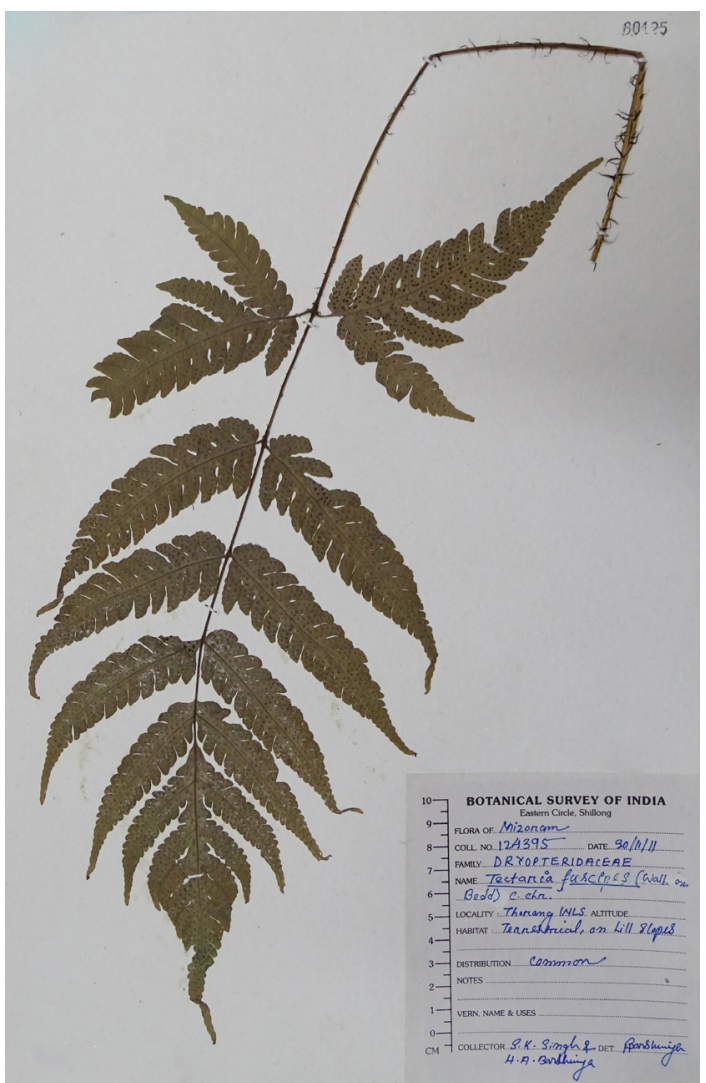

Image 38. Tectaria fuscipes 


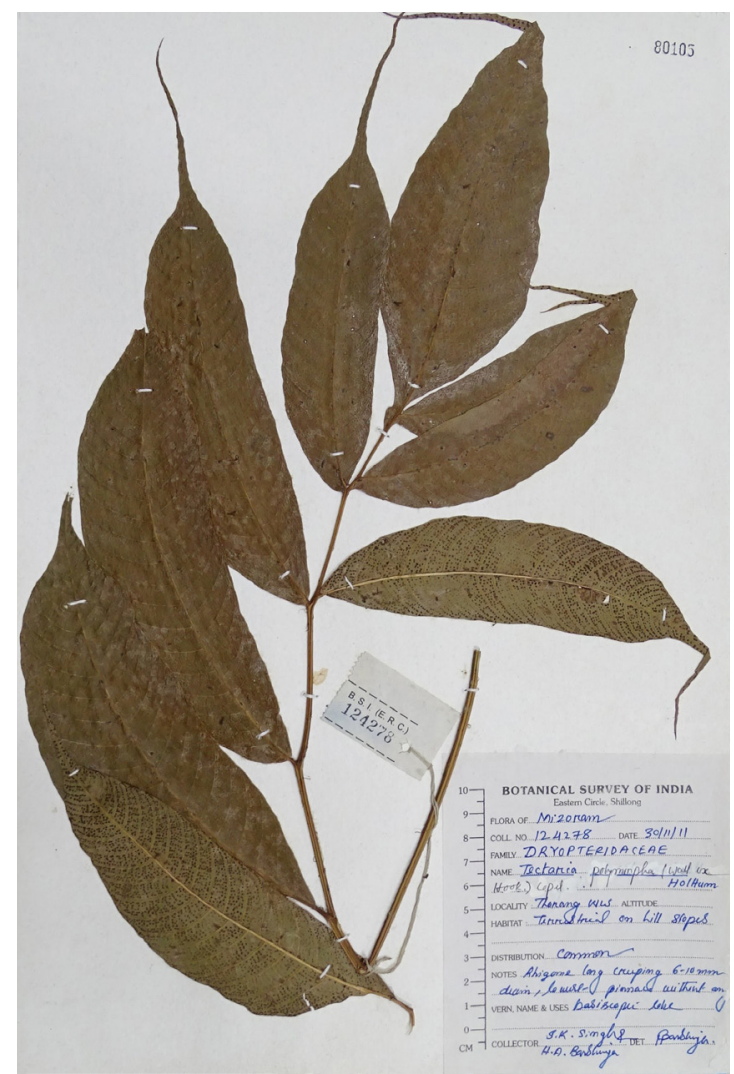

Image 39. Tectaria polymorpha

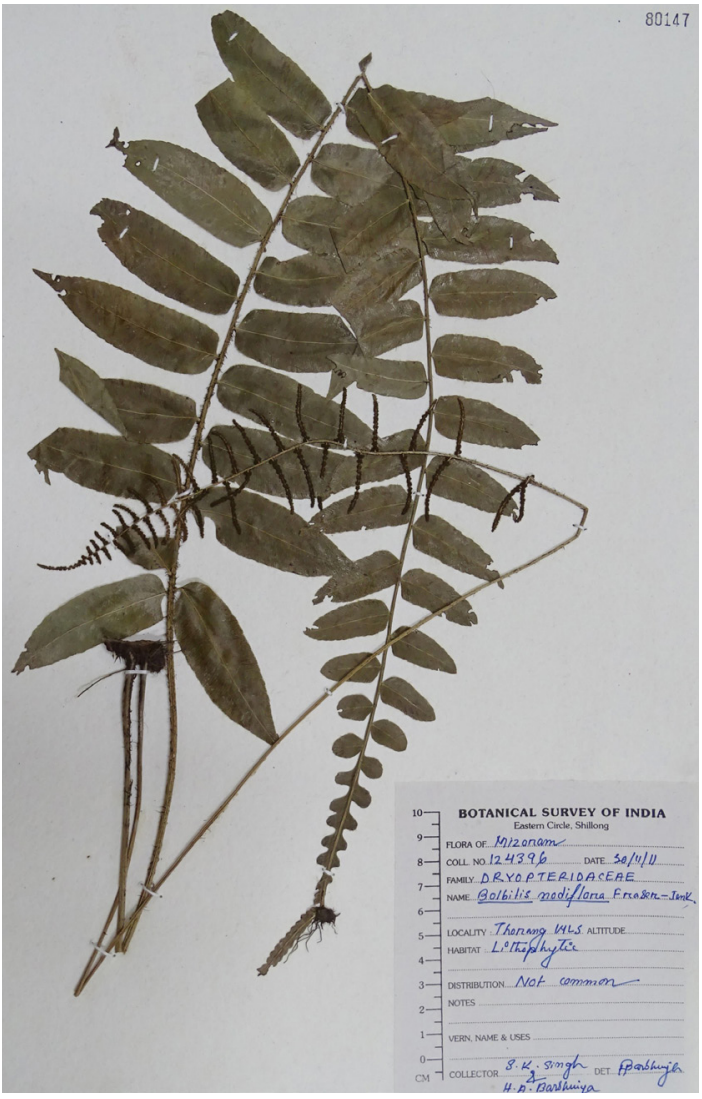

Image 41. Bolbitis nodiflora

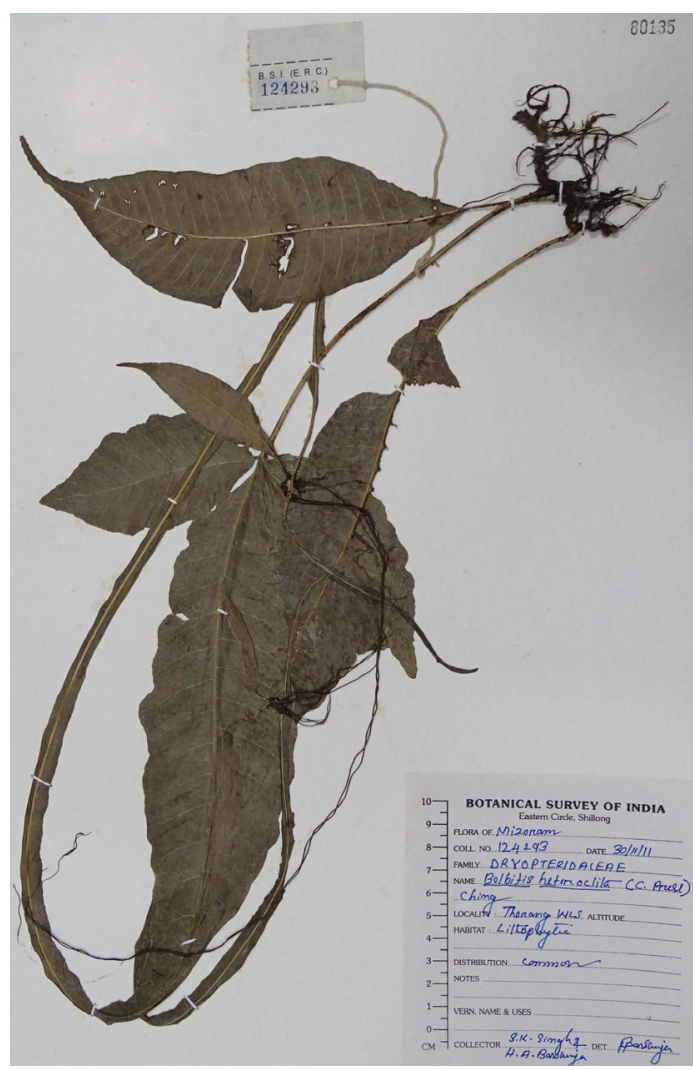

Image 40. Bolbitis heteroclita

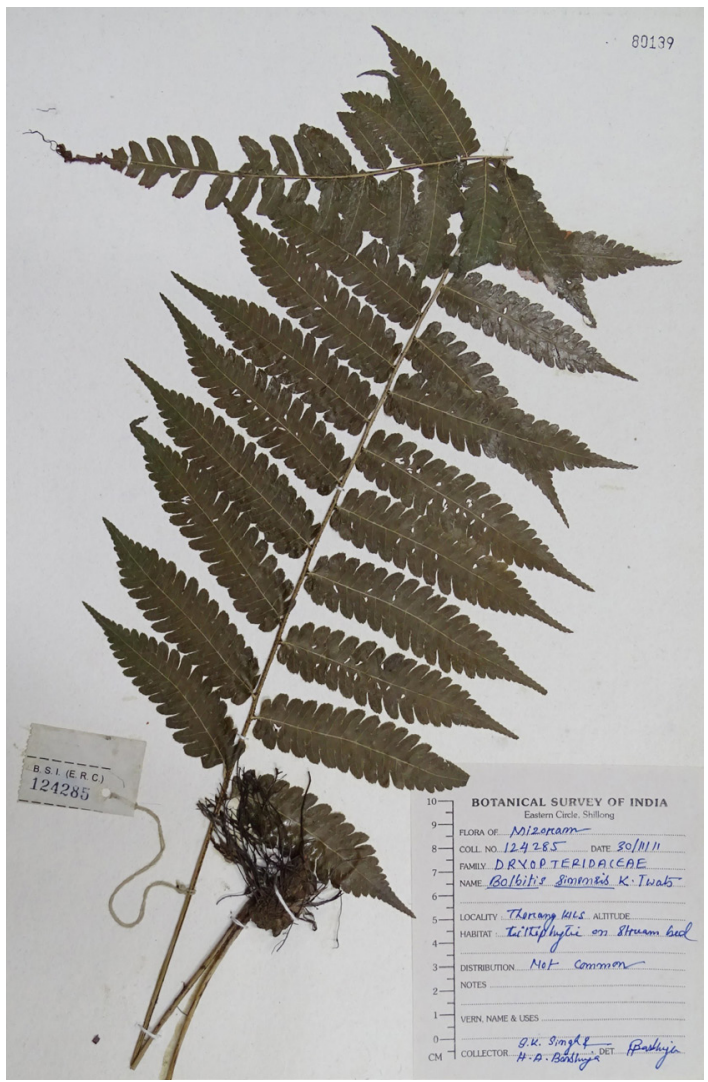

Image 42. Bolbitis sinensis 


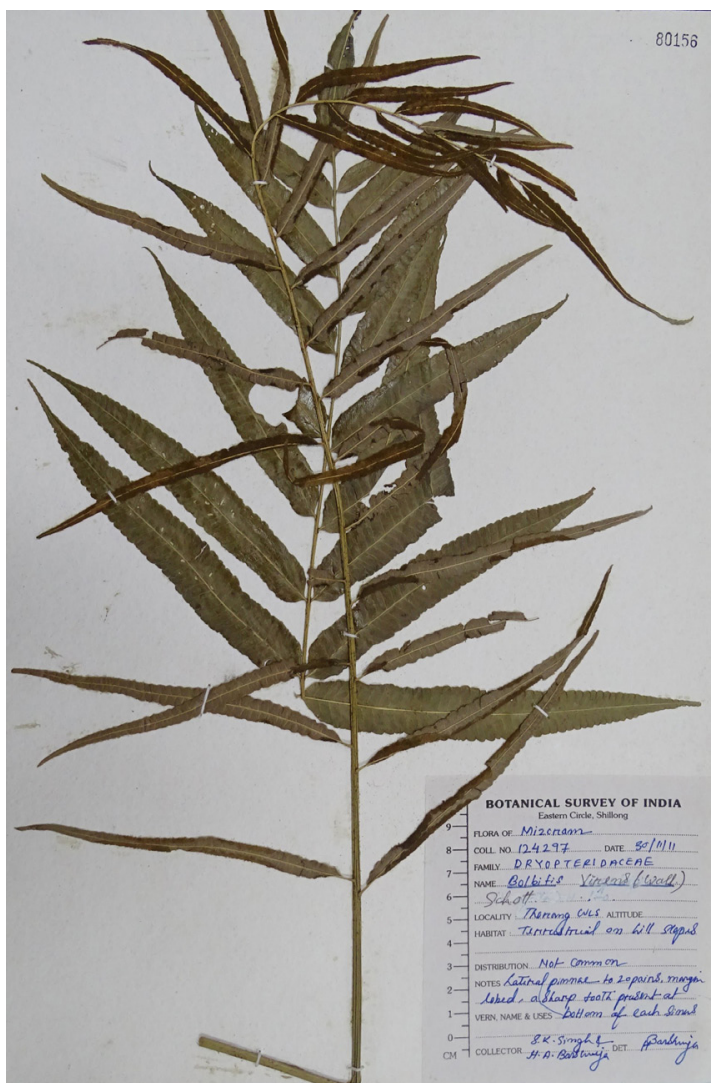

Image 43. Bolbitis virens

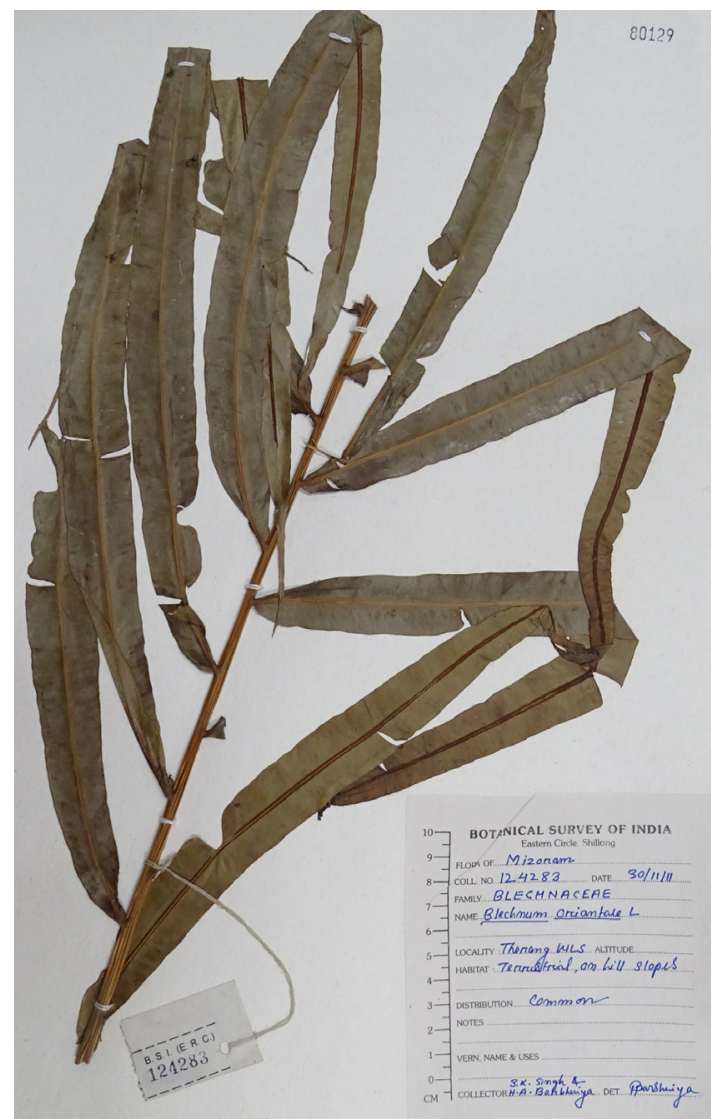

Image 44. Blechnum orientale 